\title{
Computing Value from Quality and Quantity in Human Decision-Making
}

\author{
(Di)Archy 0. de Berker, ${ }^{1,2,3,4}$ Zeb Kurth-Nelson, ${ }^{2,5}$ Robb B. Rutledge, ${ }^{1,2}$ (D) Sven Bestmann, ${ }^{1,3}$ and (Daymond J. Dolan $^{1,2}$ \\ ${ }^{1}$ Wellcome Trust Centre for Neuroimaging, University College London, London WC1N 3BG, United Kingdom, ${ }^{2}$ Max Planck University College London \\ Centre for Computational Psychiatry and Ageing Research, London WC1B 5EH, United Kingdom, ${ }^{3}$ Sobell Department of Motor Neuroscience \\ and Movement Disorders, University College London, London WC1N 3BG, United Kingdom, ${ }^{4}$ Element AI, Montreal, Quebec H2W 2R2, Canada, \\ and ${ }^{5}$ DeepMind, London EC4 3TW, United Kingdom
}

How organisms learn the value of single stimuli through experience is well described. In many decisions, however, value estimates are computed "on the fly" by combining multiple stimulus attributes. The neural basis of this computation is poorly understood. Here we explore a common scenario in which decision-makers must combine information about quality and quantity to determine the best option. Using fMRI, we examined the neural representation of quality, quantity, and their integration into an integrated subjective value signal in humans of both genders. We found that activity within inferior frontal gyrus (IFG) correlated with offer quality, while activity in the intraparietal sulcus (IPS) specifically correlated with offer quantity. Several brain regions, including the anterior cingulate cortex (ACC), were sensitive to an interaction of quality and quantity. However, the ACC was uniquely activated by quality, quantity, and their interaction, suggesting that this region provides a substrate for flexible computation of value from both quality and quantity. Furthermore, ACC signals across subjects correlated with the strength of quality and quantity signals in IFG and IPS, respectively. ACC tracking of subjective value also correlated with choice predictability. Finally, activity in the ACC was elevated for choice trials, suggesting that ACC provides a nexus for the computation of subjective value in multiattribute decision-making.

Key words: decision; number; value

\section{Significance Statement}

Would you prefer three apples or two oranges? Many choices we make each day require us to weigh up the quality and quantity of different outcomes. Using fMRI, we show that option quality is selectively represented in the inferior frontal gyrus, while option quantity correlates with areas of the intraparietal sulcus that have previously been associated with numerical processing. We show that information about the two is integrated into a value signal in the anterior cingulate cortex, and the fidelity of this integration predicts choice predictability. Our results demonstrate how on-the-fly value estimates are computed from multiple attributes in human value-based decision-making.

\section{Introduction}

Convergent evidence from human fMRI (O’Doherty et al., 2001; Montague and Berns, 2002; Kable and Glimcher, 2007; Knutson

Received March 18, 2018; revised Sept. 20, 2018; accepted Sept. 26, 2018.

Author contributions: A.O.d.B., Z.K.-N., R.B.R., S.B., and R.J.D. designed research; A.O.d.B., Z.K.-N., and R.B.R. performed research;A.O.d.B., Z.K.-N., R.B.R., and S.B. contributed unpublished reagents/analytic tools; A.O.d.B. and Z.K.-N. analyzed data; A.O.d.B., Z.K.-N., R.B.R., S.B., and R.J.D. wrote the paper.

A.0.d.B. was supported by a MRC Studentship. R.J.D. received a Wellcome Trust Senior Investigator Award (098362/Z/12/Z). The UCL-Max Planck Centre is a joint initiative supported by UCL and the Max Planck Society. The Wellcome Trust Centre for Neuroimaging is supported by core funding from the Wellcome Trust (091593/Z/10/Z). R.B.R. is supported by an MRC Career Development Award (MR/N02401X/1). We thank Laurence Hunt and Erie Boorman for fruitful discussion.

The authors declare no competing financial interests.

Correspondence should be addressed to Archy 0. de Berker, Element Al, 6650 Rue Saint-Urbain \#500, Montréal

QCH2S3G9, Canada. E-mail: archy.deberker@gmail.com.

https://doi.org/10.1523/JNEUROSCI.0706-18.2018 et al., 2007; FitzGerald et al., 2009; Levy et al., 2011) and nonhuman primate recordings (Schultz et al., 1997; Padoa-Schioppa and Assad, 2006; Hayden et al., 2011; Kennerley et al., 2011; Padoa-Schioppa and Schoenbaum, 2015) suggest that neural representations of subjective value are present in a wide variety of brain areas, potentially represented in an automatic fashion invariant to the task at hand (Lebreton et al., 2009; Grueschow et al., 2015). These value estimates are thought to provide input to value-comparison mechanisms to enable an appropriate decision between options (Padoa-Schioppa and Assad, 2006, 2008; PadoaSchioppa, 2011; Xie and Padoa-Schioppa, 2016), a process variCreative Commons Attribution 4.0 International, which permits unrestricted use, distribution and reproduction in any medium provided that the original work is properly attributed. 
ously characterized as evidence accumulation (Krajbich et al., 2010; De Martino et al., 2013; Polanía et al., 2014) or mutually inhibitory competition (Wang, 2008; Hunt et al., 2012; Chau et al., 2014). Representations of stimulus value also play a crucial role in reinforcement learning, where discrepancies between experienced and expected values give rise to the prediction errors that drive learning (Schultz et al., 1997; Sutton and Barto, 1998; Pessiglione et al., 2006; Rutledge et al., 2010; Kahnt et al., 2011).

Despite abundant and consistent evidence for value representations in specific brain areas, we still know little about how they come about and are integrated across multiple attributes. Efforts to isolate value signals within a neuroeconomic framework have used carefully controlled stimulus characteristics and action requirements in an effort to disambiguate value from its components (O'Doherty, 2014; Hunt et al., 2015). However, in the real world, we often need to construct valuations of never-before-seen objects. Recent studies using foraging tasks have emphasized that a more ethological contextualization of decision-making provides a richer account of the computations which underlying choice (Cisek and Kalaska, 2010; Kahnt et al., 2011; Chau et al., 2014; Kolling et al., 2014), highlighting a need to understand the individual component processes that contribute to value estimation. In this experiment, we drew inspiration from such foraging tasks to ask how current option value is constructed from two component parts, quality and quantity.

We designed an experiment where participants integrated information about the quality of a giftcard (how subjectively valuable it was for them to be able to spend money at a particular store) and its quantity (how much money was on the giftcard). In a behavioral session, we characterized the combination of quality and quantity to form integrated values using an auction procedure [Becker-DeGroot-Marschak (BDM); Becker et al., 1964], allowing us to select giftcards with distinct qualities. In the subsequent fMRI experiment, participants evaluated a series of individual giftcards without any choice requirement, allowing us to examine correlates of quality, quantity, and value that were uncontaminated by decision-related signals (Hunt et al., 2015).

We found that quality was represented in the inferior frontal gyrus (IFG), extending into the lateral prefrontal cortex (PFC). Conversely, quantity was associated with increasing activity in the bilateral intraparietal sulcus (IPS). To identify regions in which the two might be interacting in a manner consistent with the calculation of value, we formulated an explicit interaction term. This interaction term captures the fact that an extra unit of money on the highest quality giftcard is more valuable to the subject than an extra unit on the low-quality giftcard; you would rather have another dollar to spend at a shop you really like than
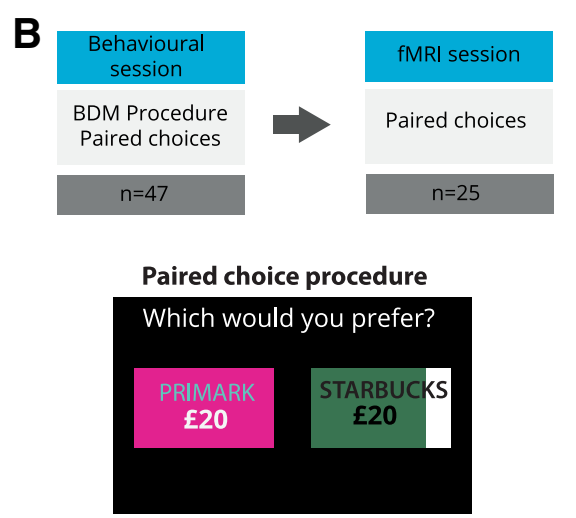

Valuation

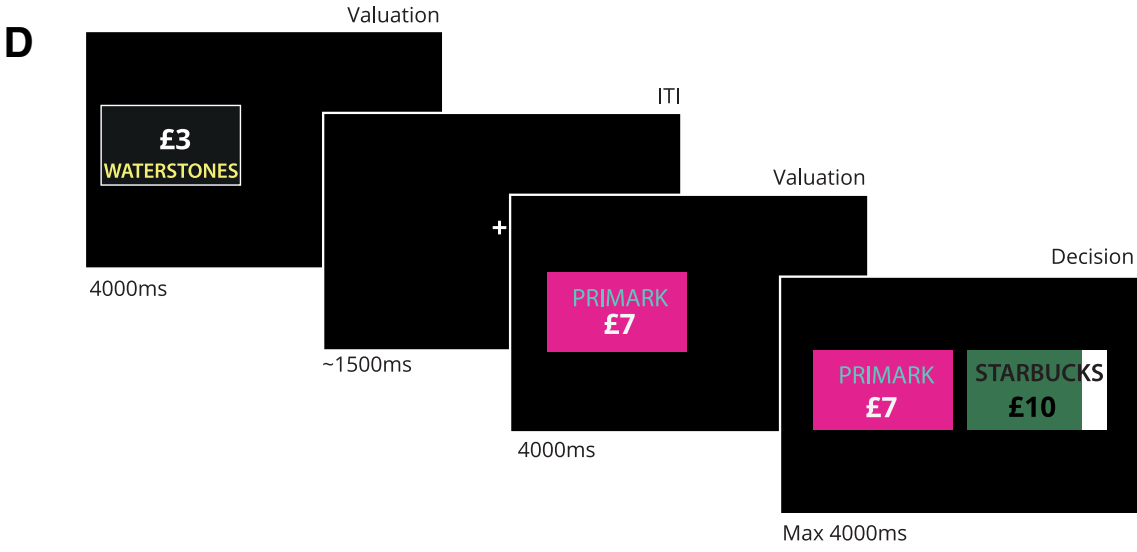

Max 4000ms

Figure 1. Experimental procedure. $\boldsymbol{A}$, We used giftcards to manipulate quality and quantity. Cards from different shops had different qualities, depending upon the subjective value of money that can be spent at that shop alone. Quantity varied as the amount of money (number of $£$ ) depicted on the card. $\boldsymbol{B}$, Following an initial behavioral session in which we mapped value

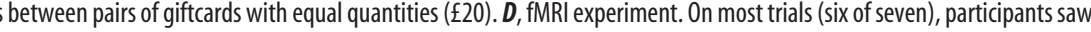

at one you dislike. This interaction (higher slope of quantity coding with higher quality) correlated with activity in the posterior cingulate cortex and bilateral superior temporal regions. Anterior cingulate cortex (ACC) displayed a conjunction of all three effects, indicative of a substrate for the calculation of integrated subjective value from its component parts. In keeping with this, we also observed repetition suppression (RS) for integrated subjective value in the cingulate cortex, with activity covarying with the absolute difference in value between stimuli presented in consecutive trials.

\section{Materials and Methods \\ Participants}

Forty-seven participants ( 25 males) participated in the behavioral study, with 26 returning for an fMRI session. Of these, one participant failed to complete the experiment due to ill health, leaving 25 participants in total for the imaging study. Both studies were approved by a local ethics committee (Research Ethics Committee UCL, reference 3450/002). Based on pilot experiments, we selected 13 giftcards that were well known to the participant population, maximized between-subject variability, and displayed minimal correlations between cards (i.e., preferences for a given card could not be predicted from preferences for other cards).

During the behavioral session, participants completed the following two tasks: an auction procedure, from which they could obtain a mixture of up to $£ 20$ cash and a $£ 20$ giftcard, and a session of paired choices 
between cards worth $£ 20$ (Fig. 1). One trial was randomly selected across both sessions and reimbursed appropriately. For the fMRI experiment, participants first completed paired choices between cards worth $£ 20$ outside of the scanner, and subsequently chose between cards worth $£ 1-20$ within the scanner. One trial from each task was reimbursed, in addition to a $£ 20$ flat rate for experiment completion.

\section{Experimental design and statistical analyses \\ Behavioral session}

Participants first performed an auction task (BDM) designed to elicit the subjective valuation of different giftcards holding varying amounts of money (Becker et al., 1964). Briefly, the BDM involves players placing a minimum bid for an item on each trial. After the experiment, a single trial is randomly selected for reimbursement. For that trial, a randomly drawn number - the "cost" - is compared with the bid. If the cost is higher than the bid, the player retains their endowment and does not receive the item. If the bid is higher than the cost, then the player receives the item, but, crucially, pays the cost rather than their bid. This removes an incentive to place low bids, resulting in an optimal strategy whereby players report their true values. Each of 13 giftcards were presented in association with 12 different quantities, giving a total of 156 trials. Following the auction task, participants chose between pairs of giftcards of matched quantity $(£ 20)$. Each combination of cards was presented twice, yielding 325 trials after the removal of trials involving two copies of the same card.

We selected a subset of participants to complete the scanning part of the study. Selection was based upon reliability, stability, and diversity of preferences over giftcards. We fit linear regressions to values reported during the auction procedure, yielding the following equation for each giftcard:

$$
\text { Integrated Value }=\beta{ }^{\star} \text { Quantity }+C \text {, }
$$

where $\beta$ and $C$ (an intercept term) were fit using robust regression. The $\beta$ values thus obtained are a measure of the quality of a giftcard, the value of a single unit of currency on that giftcard. We next assessed how well these $\beta$ values predicted paired choice (see Fig. 3), selecting subjects for whom there was a close relationship.

For the scanning session, we selected three giftcards, which were chosen to maximize variance in quality (see Fig. $3 C$ ). We thus selected the lowest and highest quality card $(\max \beta$ and $\min \beta$ ), and one closest to the mean of the two. Having performed this selection procedure, we verified that choices of these cards in the paired-choice session reflected the rankings calculated from the BDM (see Fig. 3). These $\beta$ values were used as indicators of quality for the fMRI analyses in which parametric modulators were used [general linear model (GLM) 2 and GLM3; see below].

\section{fMRI task}

The task design allowed us to examine representations of quality, quantity, and their interaction using both linear analyses and measures of repetition suppression. To avoid measurements being confounded by variables related to the dynamics of stimulus comparison (Hunt et al., 2015), on the majority of trials we presented a single stimulus (Fig. 1D) and asked participants to evaluate its desirability. The presentation side was flipped every 10 trials. Stimuli remained onscreen for $4000 \mathrm{~ms}$ before being followed by an intertrial interval (ITI; normally distributed at 1500 $\mathrm{ms}$ ) or, in one of seven trials, the appearance of a second giftcard. Participants were asked to make a choice between the two within $4000 \mathrm{~ms}$, using a button box. Failure to register a choice within this time period resulted in a "TIME OUT" message, and participants were informed before scanning that if a timed-out trial was selected for reimbursement, they would receive no payment for that part of the experiment. Each giftcard displayed in the scanner was pseudocolored red or blue to reduce gross visual differences between cards.

Repetition suppression in fMRI effects can show sensitivity to expectation (Summerfield et al., 2008), necessitating counterbalancing of stimulus order. We designed our trial presentation order such that there was no relationship between the current trial and the next one. This served the dual purposes of avoiding potential confounds in our repetition suppression analysis, and ensuring consistent engagement of our subjects, who were unable to predict when they might have to make a decision.

We defined seven trial types (red and blue versions of each three giftcards + decision trials) and used a genetic algorithm to find a stimulus (stim) order in which $p\left(\operatorname{stim}_{2}^{i} \mid \operatorname{stim}_{1}^{j}\right)$ was matched for all stimuli $i$ and $j$. We manually removed trials on which decisions were repeated, leaving a sequence of 97 stimuli. The quantity (1-20) on the giftcards was randomized, effectively orthogonalizing quality and quantity (mean correlation coefficient across participants $=0.0074, p=0.40$ ). Participants completed four runs of the task, yielding a total of 340 stimulus evaluation trials and 48 decision trials.

\section{Logistic regression modeling of choices during fMRI task}

We used logistic regression to characterize the factors modulating choices in the scanner. For each participant, we fit a model to predict whether they chose the new card (presented during the decision trial) or the old card (on-screen from the valuation trial):

\section{Choice $(t)$}

$$
=s\left(\beta_{0}+\beta_{0} \text { Quality }_{\text {New-Old }}+\beta_{2} \text { Quantity }_{\text {New-Old }}+\beta_{3} \text { Interaction }_{\text {New-Old }}\right),
$$

where $\beta_{0}$ is a constant term accounting for option-independent biases in choice, $\beta_{1-3}$ are regression coefficients describing the effect of each term on choice, and $s$ is the sigmoid function:

$$
s(x)=\frac{1}{1+e^{-x}} .
$$

Quality was defined using the $\beta$ values from the BDM auction (see above; see Fig. $3 A$ ), while quantity merely reflected the monetary amount (in pounds) depicted on each giftcard. We formulate the interaction term by first normalizing quality and quantity, and then taking the product.

To assess choice predictability, we took the output of the model (valued between 0 and 1 ), rounded it (such that choices were either a 0 or a 1 ), and compared it to the vector of actual choices made by the participant. Predictability was then defined simply as the percentage of choices correctly predicted by the model.

\section{fMRI data acquisition}

Data were acquired using a Siemens $3 \mathrm{~T}$ Trio Scanner with a 32-channel head coil at the Wellcome Trust Centre for Neuroimaging. We used a 2D echoplanar image (EPI) sequence optimized to minimize dropout in the orbitofrontal cortex (OFC; Weiskopf et al., 2006), with voxels $3 \mathrm{~mm}$ isotropic ( $\mathrm{TR}=3.36 \mathrm{~s}, \mathrm{TE}=30 \mathrm{~ms})$, with 48 slices giving whole-brain coverage. Slices were tilted at $-30^{\circ}$. Scans were preceded by a field map $(\mathrm{TE} 1=10 \mathrm{~ms}, \mathrm{TE} 2=12.46 \mathrm{~ms})$. The first five volumes of each run were discarded to allow for T1 equilibration. We also acquired a T1-weighted structural scan for each subject, comprising 176 slices over a field of view of $256 \mathrm{~mm}$ with a $1 \mathrm{~mm}$ isotropic resolution $(\mathrm{TR}=7.92 \mathrm{~ms}$, $\mathrm{TE}=2.48$ ms; Deichmann et al., 2004). Throughout scanning, we monitored the breathing rate, using a pneumatic belt and pulse, and blood oxygenation, using an infrared pulse oximeter (Model 8600 F0, NONIN). Both were digitized and recorded via Spike2 (version 6.17), and subsequently included in GLM analyses of brain activity along with regressors derived from motion correction (Hutton et al., 2011).

\section{fMRI data preprocessing}

All preprocessing and data analysis took place in SPM12 (http://www. fil.ion.ucl.ac.uk/spm/). Subsequent data visualization took place in MRIcron (http://people.cas.sc.edu/rorden/mricron/index.html) and MRIcroGL (http://www.cabiatl.com/mricrogl/). Having discarding the first 5 volumes, we corrected EPIs for field inhomogeneities using acquired field maps. We then bias corrected, slice-time corrected (to the middle slice), and realigned and unwarped to the first EPI for each participant. EPIs were then co-registered to each participant's structural scan. EPIs were then coregistered to each participant's structural scan. We used the DARTEL toolbox for between-subject registration and normalization (Ashburner, 2007). Structural images were first segmented into white matter, gray matter, and CSF components. Segmented images were then iteratively warped into normalized MNI space, providing a 
template that was then used to normalize EPIs, a step that included Gaussian smoothing at $8 \mathrm{~mm}$ FWHM.

\section{fMRI data analysis}

Data were analyzed using a series of GLMs. These were estimated for each participant, including the calculation of contrasts between different regressors (first-level analysis). This provided summary statistics ( $\beta$ values) that could be tested at a population level versus a null hypothesis that they were on average equal to zero (second-level analysis; Friston et al., 1999). To obviate multiple comparisons when performing whole-brain analyses, we applied a correction using a cluster-defining threshold of $p<$ 0.005 , and a cluster-corrected familywise error (FWE) threshold of $p<$ 0.05 , except in the analysis of repetition suppression (GLM2, below), where a more lenient cluster-forming threshold of $p<0.01$ was used, which is in line with recent repetition suppression studies (Barron et al., 2013; Garvert et al., 2015; Boorman et al., 2016). To extract the parameter estimates displayed in Figure 5, we used group-functional ROIs thresholded at $p<0.005$. For the conjunction analysis described in Figure 5, we took the product of three binary masks (quality, quantity, and interaction), each thresholded at $p_{\text {uncorrected }}<0.05$, resulting in an FWE rate of $p_{\text {uncorrected }}=0.000125$.

GLM1: quality and quantity. Our first GLM incorporated separate onset regressors for cards of different qualities (low, medium, and high). Each of these was modeled as a 4-s-long boxcar and was associated with a parametric modulator corresponding to the quantity on the card at each presentation. We used a fourth-onset regressor corresponding to decision trials, which were modeled as $\Delta$ functions. This GLM was used to perform a whole-brain analysis of value computations during evaluation trials.

We performed the following three key contrasts:

$$
\begin{gathered}
\text { Quality: }\left[\text { Quality } \text { High }- \text { Quality }_{\text {Low }}\right. \text { ] } \\
\text { Quantity: [Quantity } \text { HighQuality }- \text { Quantity }_{\text {MediumQuality }}+\text { Quantity }_{\text {LowQuality }} \text { ] } \\
\text { Interaction: [Quantity } \\
\text { HighQuality } \\
- \text { Quantity }_{\text {LowQuality }} \text { ]. }
\end{gathered}
$$

The interaction analysis was constructed to test for regions displaying steeper coding of quantity for high-quality cards compared with lowquality cards, consistent with value integration. This corresponds to an intuition that an extra unit of a more desirable good (e.g., a Ferrari) is worth more than an extra unit of a less desirable good (e.g., an apple). We excluded trials preceding decisions from the evaluation regressors to guard against contamination of the evaluation regressors by decisionrelated activity, a possibility arising out of the lack of ITI between evaluation and decision trials.

GLM2: repetition suppression. Following the numerosity-coding literature (Piazza et al., 2004, 2007; Jacob and Nieder, 2009), we designed a repetition-suppression analysis based upon the absolute change in value between trials (see Fig. 7A). We used this analysis to reveal repetition suppression effects within ROIs identified by the whole-brain analysis using GLM1.

$\Delta$ IntegratedValue $(t)=\mid$ IntegratedValue $(t)-\operatorname{IntegratedValue}(t-1) \mid$.

where IntegratedValue $(t)$ is simply the product of quality and quantity on trial $t$. We used a single-onset regressor to represent all giftcard presentations, again using a $4 \mathrm{~s}$ boxcar, with parametric modulators for $\Delta$ Integrated Value, and, as a precaution, Integrated Value. The inclusion of Integrated Value in the model allowed us to confirm that the effects of $\Delta$ Integrated Value were not simply the result of spurious correlation with Integrated Value itself. Trials following decisions were excluded as they were preceded by a pair of stimuli, obfuscating the calculation of stimulus similarity. As before, we used a second-onset regressor for decision trials. Contrasts were calculated merely as the value of the relevant parametric modulators.

GLM3: integrated value. To obtain a measure of integrated value coding, we used a single $4 \mathrm{~s}$ boxcar for all evaluation trials, which was associated with a parametric modulator for integrated value (Quality $\times$ Quantity), excluding predecision trials as in GLM1. As in GLM1 and 2, decision trials were modeled in a separate regressor with $\Delta$ onsets. We used this analysis within ROIs identified by the whole-brain analysis in GLM1, to confirm that the ACC region showing a conjunction of quality, quantity, and interaction effects could also be described as codingintegrated value.

Statistical tests. Parameter estimates from fMRI are normally distributed, permitting the use of parametric statistics ( $t$ tests and Pearson correlations). When analyzing distributions that we knew a priori to be non-normal (e.g., predictability, which is bounded at 0 and 100), we used nonparametric equivalents (sign tests and Spearman rank coefficients). All statistical testing was performed in Matlab.

\section{Results}

Behavioral session establishes stable quality estimates

We used a behavioral session to identify participants for whom we could find giftcards with consistently different subjective qualities (Fig. 1B). The behavioral session consisted two tasks. Participants $(n=47)$ performed a BDM auction (Becker et al., 1964) and a series of paired choices, each involving a selection of 13 giftcards (Fig. $1 C$ ). In the BDM auction, players reported how much they would be willing to pay for a giftcard loaded with a certain amount of money, from $\mathfrak{E} 1$ to $\mathfrak{E} 20$. Subsequently, participants made paired choices between different giftcards containing matched sums $(\mathfrak{2} 20)$.

We used a linear fit to the relationship between the amount of money on the giftcard and the amount bid for each giftcard during the BDM to provide a measure of the quality of each giftcard for each participant (Fig. 2A). To maximize power in the fMRI study, we selected subjects whose bids were predictable (Fig. 2A) and for whom we could select three giftcards with distinct qualities (Fig. 2B, circled points, $C$ ). By way of confirmation that BDM-estimated values predicted choice, we next compared quality estimates from the BDM with the number of choices of each giftcard in the paired-choice session, preferring subjects for whom there was a high correlation (Fig. 2B).

Selected subjects thus displayed consistent BDM bids, a high correlation between preferences elicited in the BDM and pairedchoice sessions, and a low maximum correlation among quality, quantity, and integrated value (Fig. 3). Integrated value, calculated as the product of quality and quantity, effectively provided a prediction of the bid a participant would place for a given giftcard. The correlation among quality, quantity, and integrated value reflects the diversity of giftcard qualities. Giftcards with disparate qualities limit the correlation between quantity and integrated value (Fig. $2 C$, $i$, iii), while if all giftcards have similar qualities, the quantity/integrated value correlation will be high (Fig. 2C, ii).

\section{fMRI experiment: subjects integrate quality and quantity in choice}

For each participant in the fMRI experiment $(n=25)$, we used data from the behavioral session to select the following three giftcards: the giftcard that displayed the steepest relationship between BDM bid and quantity (high quality); the giftcard that displayed the lowest (low quality); and a giftcard of intermediate slope (medium quality; Fig. $3 A$ ). In a prescanning paired-choice session, we confirmed that preference estimates from the preceding behavioral session were stable, with subjects making choices among the three selected giftcards in a highly predictable manner (Fig. 3B).

Within the scanner, participants made choices between giftcards of varying quality and quantity on one of seven trials (Fig. $1 D)$, resulting in a total of 48 decisions. Participants remained highly engaged throughout, exceeding the time limit for choice of $4000 \mathrm{~ms}$ in only 7 of 1200 choices. We used a logistic regression 
A
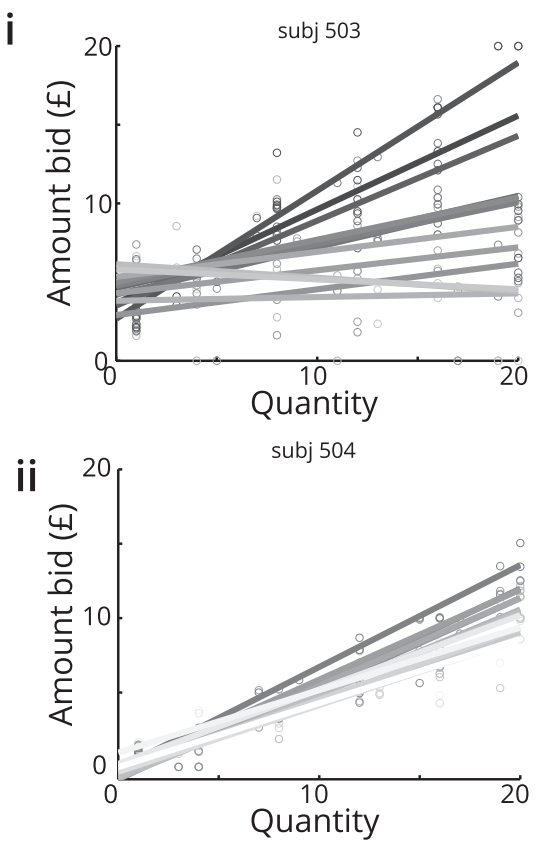

iii

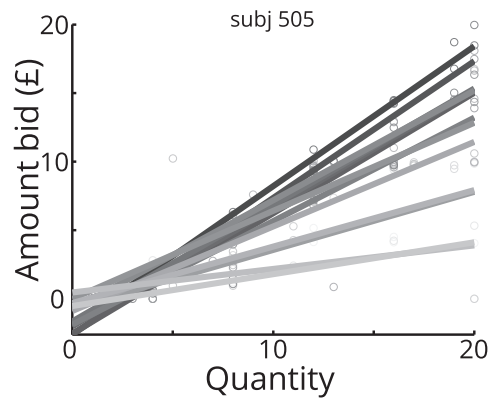

B i
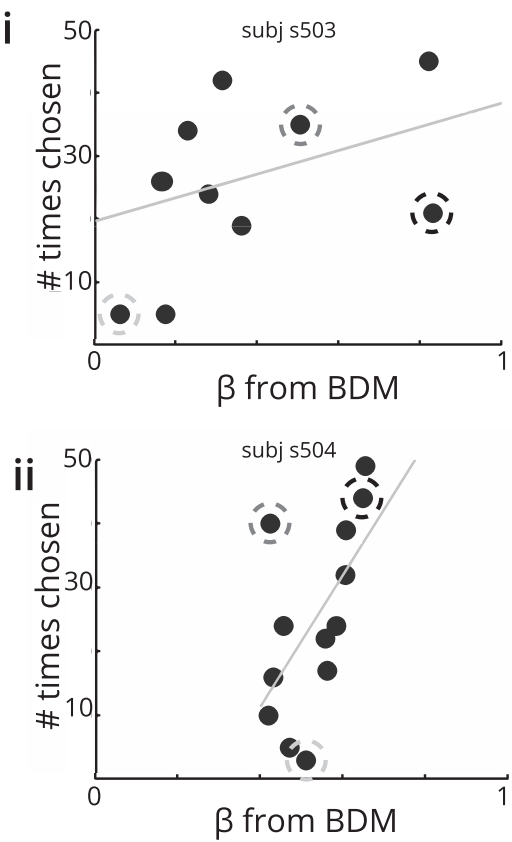

iii

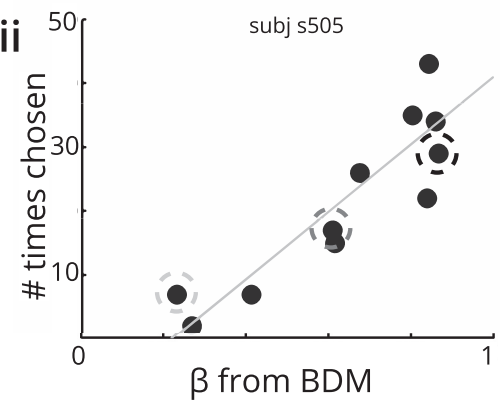

C i
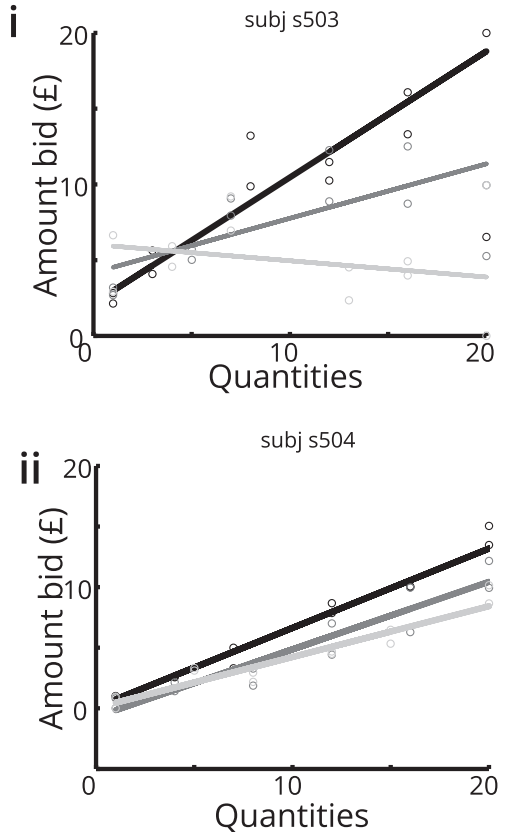

iii

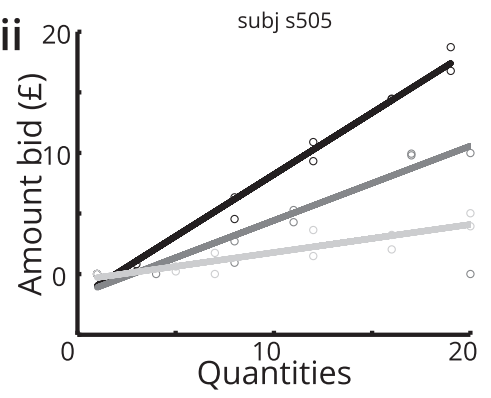

Figure 2. Example participants from behavioral experiment. We used data from the behavioral session to determine subsequent inclusion in an fMRI study based on criteria of consistency and diversity of preferences for different giftcards (assessed using the predictability of BDM ratings), relationships between BDM and paired-choice tasks, and correlations between quantity and subjective value. $A$, First, we examined quantity-bid relationships for the 13 different giftcards. The slope of the quantity- bid relationship for each giftcard is a measure of that the quality of the giftcard, with higher slopes corresponding to more valuable brands. Here, the participant in $\boldsymbol{i}$ has diverse but noisy preferences, the participant in $i \boldsymbol{i}$ is consistent but has similar preferences across giftcards, while the participant in iii displays an acceptable level of consistency while maintaining diverse preferences. $\boldsymbol{B}$, To assess preference stability, we compared the slope of lines estimated from the BDM task with the number of times each giftcard was chosen in the paired-choice task. The participant in $\boldsymbol{i}$ shows a weak relationship between choices in each session; the participant in $\boldsymbol{i} \boldsymbol{i}$ is consistent but shows little variability; and the participant in iii is both consistent and displays diverse preferences. $C$, For the fMRI experiment, we selected three giftcards for each participant that differed maximally in quality. Here we show BDM plots for selected cards. As before, $i$ is noisy but shows diverse quality preferences, $i i$ has similar preferences over giftcards, and $i i i$ has consistent and diverse preferences over giftcards.

analysis to quantify the impact of differences between the two options upon choice. We calculated an interaction term as the meancentered product of quality and quantity. Intuitively, the interaction term captures the fact that an extra pound on the highest quality giftcard is more valuable to the subject than an extra pound on the low-quality giftcard. Differences among options in quality, quantity, and their interaction all influenced participants' choices (quality: $t_{(24)}=8.6, p<0.001$; quantity $t_{(24)}=13.7, p<0.001$; interaction: $t_{(24)}=3.8, p<0.001$; Fig. $3 C$ ), implying that participants combined information about quality and quantity to estimate integrated subjective value, rather than considering the two attributes independently.

\section{Brain activity associated with quality, quantity, and} their interaction

In the scanner, participants were shown a single giftcard and asked to internally evaluate it (evaluation trials), in the knowledge that they might have to make a fast decision between that option and another (decision trials; Fig. 1D). The preponderance of valuation trials (340 of 388) provided us with an opportunity to examine value computation in isolation, without potentially confounding effects of decision dynamics (Hunt et al., 2015).

To isolate elements of value representation, we used GLMs of voxelwise brain activity to examine the representation of quality, quantity, and their interaction in valuation trials. We split card presentations by quality (low, medium, high) and associated each onset with a parametric modulator corresponding to the quantity presented on that trial. This allowed us to index the main effects of card quality $_{\text {(Quality }}$ High - Quality $_{\text {Low }}$, card quantity (Quantity LowQuality $_{\text {( }}$ + Quantity $_{\text {MediumQuality }}+$ Quantity HighQuality $)$, and the interaction between the two (Quantity HighQuality $_{\text {- Quantity }}$ LowQuality $)$.

The interaction term allows us to identify regions where quantity affects activity more when giftcard value is high compared with when it is low. By decomposing value in this way-into quality, quantity, and their interaction-we can identify brain regions displaying specific relationships with each component, as well as regions showing an overlap of all three effects. This conjunction analysis is more stringent than a simple contrast for 
A

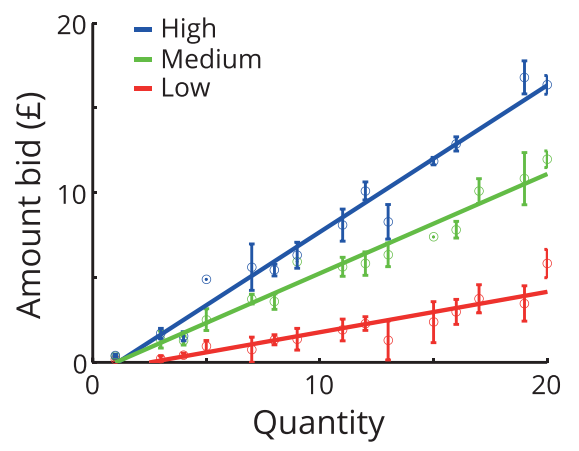

B

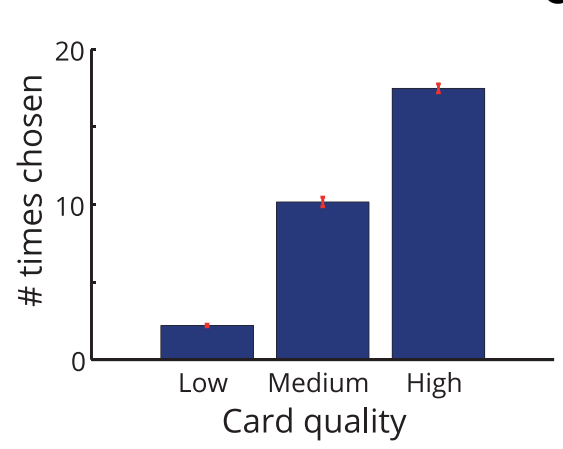

C

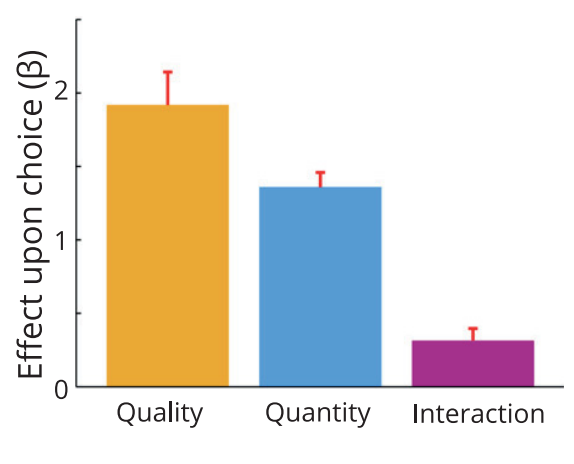

Figure 3. Behavioral results for subjects in scanning experiment. $A$, Average quantity-bid functions show the difference in quality for three selected giftcards for subjects who completed both the behavioral and fMRI sessions ( $n=25$ ). $\boldsymbol{B}$, In a prescanning paired-choice session, we confirmed that the ordering of cards by quality was highly consistent between sessions. $\boldsymbol{C}$, Analysis of choices made in the MRI scanner. During the fMRI experiment, participants made 48 choices between cards of varying quality and quantity (Fig. 1D). We used the differences between options to predict choices using logistic regression. The differences between options in both quality $\left(t_{(24)}=8.6, p<0.001\right)$ and quantity $\left(t_{(24)}=13.7, p<0.001\right)$ were predictive of choice. Importantly, the interaction between quality and quantity also predicted choice $\left(t_{(24)}=3.8, p<0.001\right)$, consistent with the multiplicative relationship expected from the observed quantity- utility functions $(\boldsymbol{A})$. Errors bars represent the SEM across subjects.

integrated value, because it prevents erroneously identifying regions that simply have a strong correlation with only quality or quantity. Motivated by this same logic, recent studies formulate fMRI contrasts for reward prediction errors (RPEs) as a conjunction of positive coding for reward and negative coding for reward expectation, thus avoiding false-positive results arising from the correlation between RPEs and other variables such as reward itself (Rutledge et al., 2010).

We found three largely nonoverlapping patterns of response corresponding to the representation of offer quality, quantity, and their interaction. Higher card quality was associated with greater activity in bilateral IFG, centered on the pars opercularis (left: peak MNI $=-54,12,30 ; t_{(24)}=4.79, p_{\text {FWE-corrected }}=0.023$; right: peak $\mathrm{MNI}=51,9,27 ; t_{(24)}=4.37, p_{\text {FWE-corrected }}=0.032$; Fig. $4 A$ ). On the left, this extended into dorsolateral PFC (peak MNI $\left.=-36,48,24 ; t_{(24)}=3.16, p_{\text {FWE-corrected }}=0.044\right)$ and included Broca's area, an area associated with semantic comprehension (Price, 2012), arguably a process necessary for evaluating abstract stimuli such as giftcards. Parameter estimates extracted from group-level functional ROIs within IFG (defined at $p<$ $0.005)$ suggested an absence of sensitivity to either quantity $\left(t_{(24)}=1.18, p=0.24\right)$ or the interaction of quantity and quality $\left.t_{(24)}=1.53, p=0.14\right)$ in this region, although direct comparisons did not distinguish quality coding from that of quantity or the interaction (quantity: $t_{(24)}=1.88, p=0.073$; interaction: $\left.t_{(24)}=1.88, p=0.17\right)$.

Offer quantity correlated with activity in bilateral IPS (Left: peak MNI, $-27,-66,51 ; t_{(24)}=4.77, p_{\text {FWE-corrected }}<0.001$; right: peak MNI, $\left.33,-66,51 ; t_{(24)}=4.68, p_{\text {FWE-corrected }}<0.001\right)$ resonating with a role for this region in numerical reasoning in humans and nonhuman primates (Nieder and Miller, 2004; Piazza et al., 2004, 2007; Pinel et al., 2004; Harvey et al., 2013; Fig. $4 B)$. As for IFG, activity in the IPS was selective for number, with no sensitivity to quality $\left(t_{(24)}=0.19, p=0.85\right)$ or the interaction of quality and quantity $\left(t_{(24)}=0.84, p=0.41\right)$. This indicates that IPS is not performing value coding per se, but specifically represents the quantity of available options. Direct comparisons confirmed that quantity correlations were greater than those for quality $\left(t_{(24)}=3.52, p=0.0017\right)$ and for the interaction $\left(t_{(24)}=\right.$ $3.36, p=0.0025)$. We also observed quantity-related activity in bilateral visual cortex (left: peak MNI, $-33,-87,-12 ; t_{(24)}=$ 5.54, $p_{\text {FWE-corrected }}<0.001$; right: peak MNI, 27, $-87,-12$; $\left.t_{(24)}=5.49, p_{\text {FWE-corrected }}<0.001\right)$.
Finally, we asked whether activity in any region of the brain was associated with an interaction between quality and quantity, correlating more steeply with quantity for high-quality giftcards compared with low-quality giftcards. This is a signature of value computation, involving additional processing above and beyond a simple reflection of option quality or quantity. The most prominent effect was located along the posterior cingulate cortex (peak MNI, $\left.-12,-15,54 ; t_{(24)}=3.57, p_{\text {FWE-corrected }}<0.001\right)$ where activity was specific to the interaction term, with no evidence of quality $\left(t_{(24)}=-0.14, p=0.89\right)$ or quantity $\left(t_{(24)}=0.77, p=\right.$ $0.45)$ correlations, implying that despite the involvement of this region in value computation, it does not represent an integrated value signal per se. Direct comparisons confirmed that the interaction effect exceeded both quality $\left(t_{(24)}=2.93, p=0.0072\right)$ and quantity $\left(t_{(24)}=2.53, p=0.018\right)$ contrasts. Interaction contrast effects were also present in bilateral superior temporal lobes (left: peak MNI, $-63,-45,0 ; t_{(24)}=5.55, p_{\mathrm{FWE}-\text { corrected }}<0.001$; right: peak MNI, 48, $-33,3 ; t_{(24)}=4.85, p_{\text {FWE-corrected }}<0.001$; Fig. $4 C)$.

\section{Computation of integrated value from component parts in the cingulate}

Having characterized neural responses to individual components of option value (quality, quantity, and their interaction), we next asked whether any regions represented integrated value. To do so, we formulated a parametric regressor for subjective integrated value, by combining quality and quantity mutliplicatively in the manner suggested by our behavioral results (Fig. 3).

However, since integrated value is correlated with quality and quantity (though this correlation is limited by design) testing for effects of integrated value presents a problem as regions sensitive to quality or quantity alone might appear to reflect integrated value. To overcome this, we supplemented our parametric analysis with a conjunction analysis, reasoning that a region truly representing integrated value ought to display sensitivity to all of its components: quality, quantity, and their interaction. Importantly, the interaction of two mean-centered variables is decorrelated from either component, giving us a way to check for correlates of the computation of subjective value.

Both analyses revealed a striking convergence on the ACC (Fig. 5) where activity covaried with a parametric modulator for integrated value (peak MNI, $-12,21,39 ; t_{(24)}=6.04, p_{\text {FWE-corrected }}$ $<0.001$ ) and showed a conjunction of effects of quality, quantity, 
A

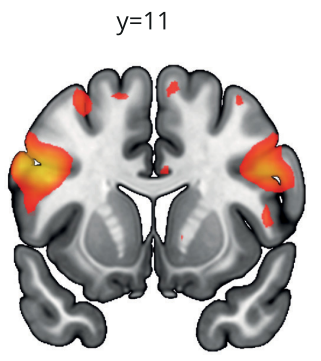

B

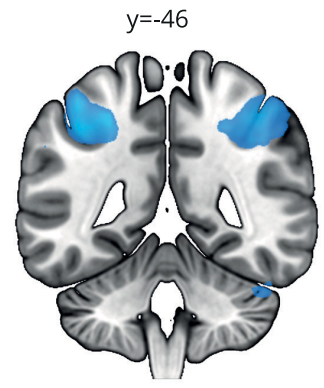

C

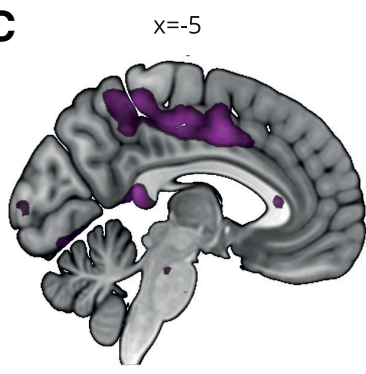

$\mathrm{z}=27$
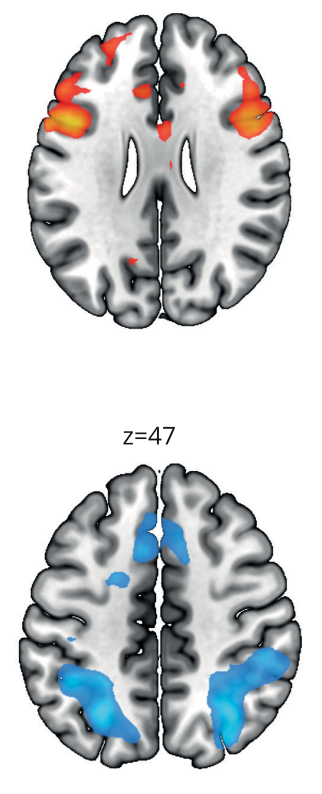
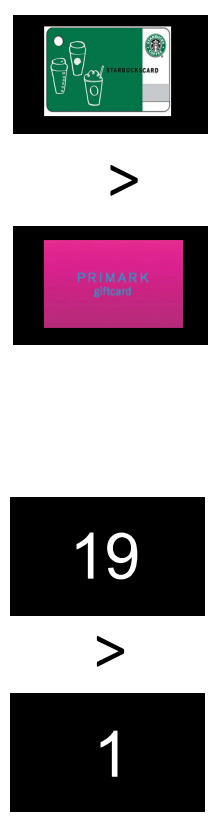

2.5

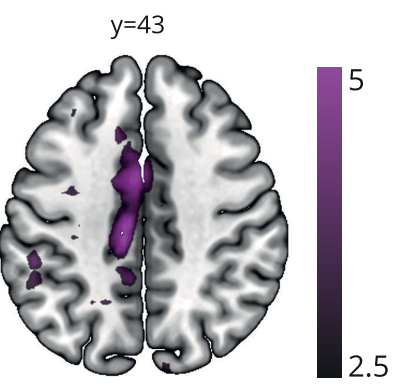

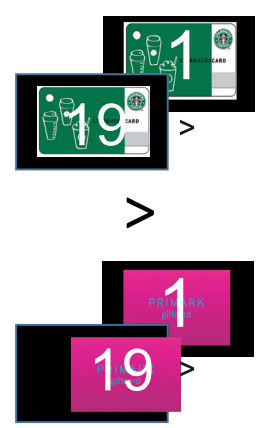
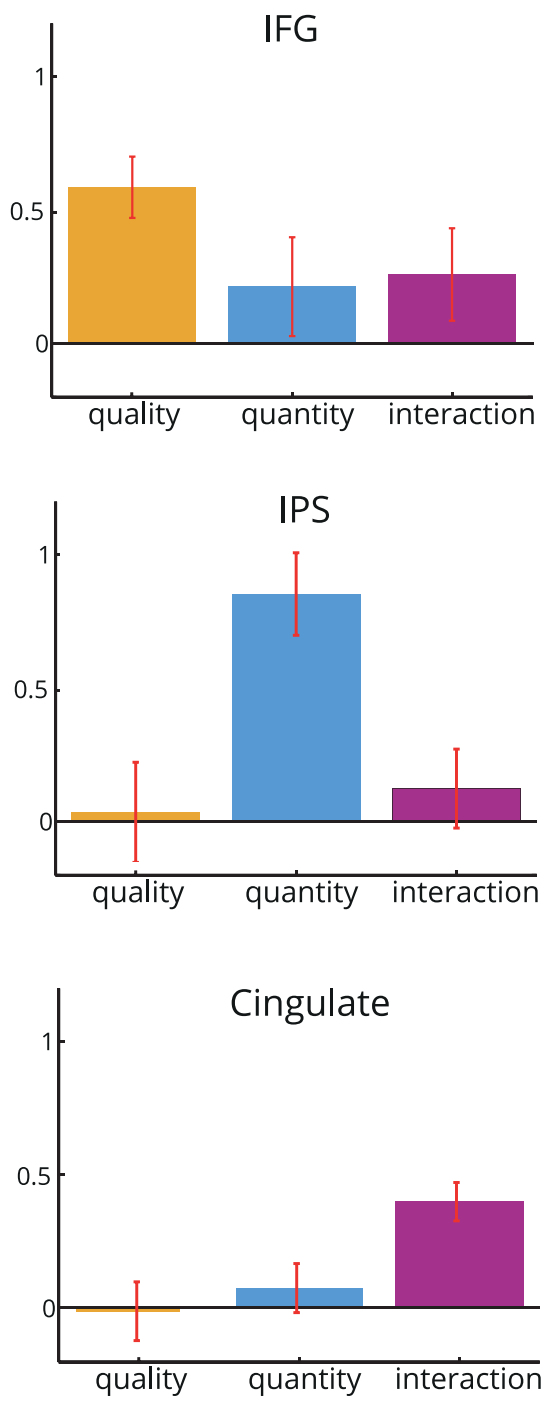

Figure 4. Representation of quality, quantity, and their interaction. $\boldsymbol{A}$, We observed bilateral coding of offer quality (Quality ${ }_{\text {High }}-$ Quality $_{\text {Low }}$ ) bilaterally in the IFG. $\boldsymbol{B}$, Increasing quantity, as tested by Quantity HighQuality + Quantity $_{\text {MediumQuality }}+$ Quantity $_{\text {LowQuality' }}$ was associated with greater activity bilaterally in the IPS. C, Activations in the posterior cingulate cortex were consistent

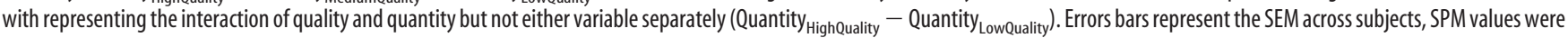
thresholded at $p<0.01$ for visualization.

and their interaction (all $p<0.05_{\text {uncorrected }}$ ). This chimes with known roles of this regions, including the fact that it contains neurons that multiplex attributes in value-based decision-making (Kennerley et al., 2011) and its necessity for value learning (Rushworth and Behrens, 2008; Hayden et al., 2009). Decomposing the interaction effect within the ACC, we observed that although quantity coding for all three qualities was positive, it was only significantly so in the high-quality condition (Quantity LowQuality: $t_{(24)}=0.15, p=0.87$; Quantity MidQuality: $_{(24)}=1.51, p=0.14$; Quantity HighQuality: $\left._{(24)}=4.24, p<0.001\right)$. By way of comparison, all three of these effects were significant in the IPS region pictured in Figure $4 B$, with no significant difference between coding of quantity for high-quality and low-quality giftcards (Quantity $_{\text {LowQuality: }} t_{(24)}=2.30, p=0.03$; Quantity MidQuality $_{(24)}=$ 2.69, $p=0.012$; Quantity HighQuality $_{(24)}=4.78, p<0.001$; Quantity $_{\text {HighQuality }}$ vs Quantity LowQuality $\left._{(24)}=1.17, p=0.25\right)$.

We next reasoned that if the ACC value estimates guide choice, then we should see greater activity in decision trials compared with valuation trials. This was indeed the case with decision trials associated with enhanced activity in the same region (peak
MNI, 9, 15, 45; $t_{(24)}=17.03, p_{\text {FWE-corrected }}<0.001$; Fig. 5B). Dorsally, this region overlaps with activity in dorsomedial PFC (dmPFC) showing an integrated value difference signal (Lebreton et al., 2009; Grueschow et al., 2015) and previously characterized as the final value comparison step before motor output (Hare et al., 2011).

Our analyses revealed dissociable representations of quality, in the IFG, and quantity, in the IPS. Although we lack the temporal precision to test whether these segregated representations precede the emergence of integrated value signals in ACC, we nevertheless can ask whether between-subject variability in component coding is related to between-subject variability in ACC representations. We found evidence that this was the case, with stronger IFG encoding of quality associated with stronger coding of quality in ACC $(r=0.63, p<$ 0.001; Fig. $5 C$ ), while stronger IPS encoding of quantity was associated with stronger quantity coding in the ACC $(r=0.68$, $p<0.001$; Fig. $5 F$ ). Importantly, the converse correlations did not hold, with parameter estimates for IGF quality unrelated 
A
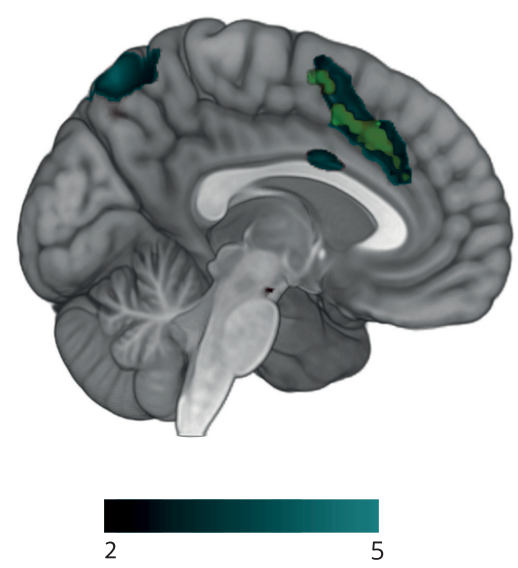

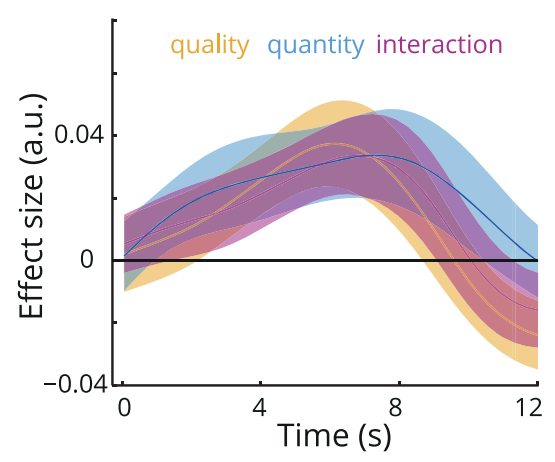

B

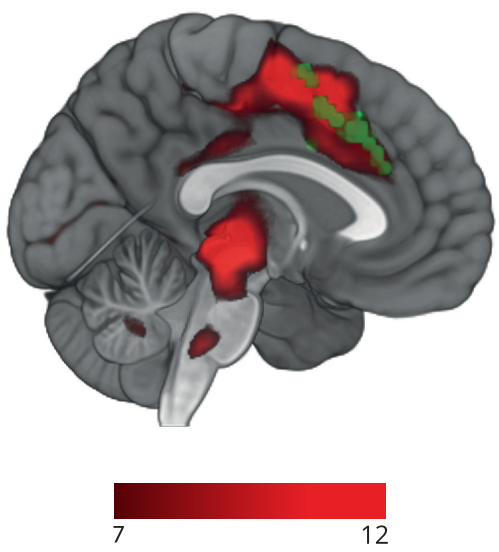

C

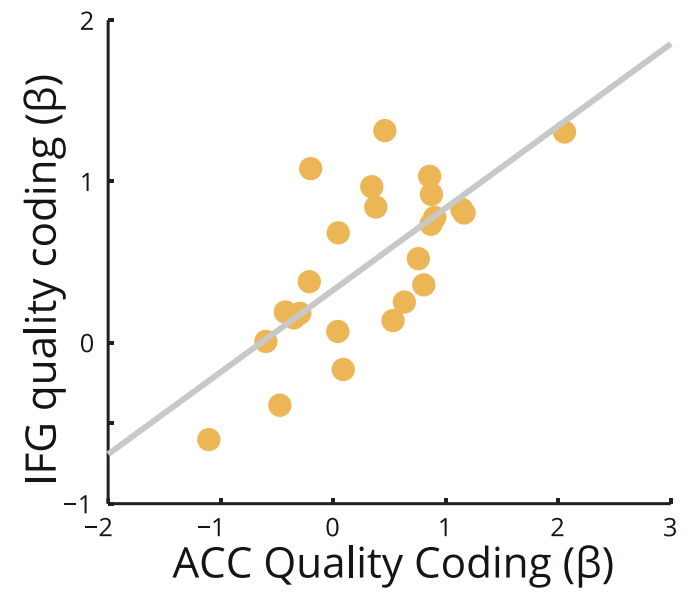

E

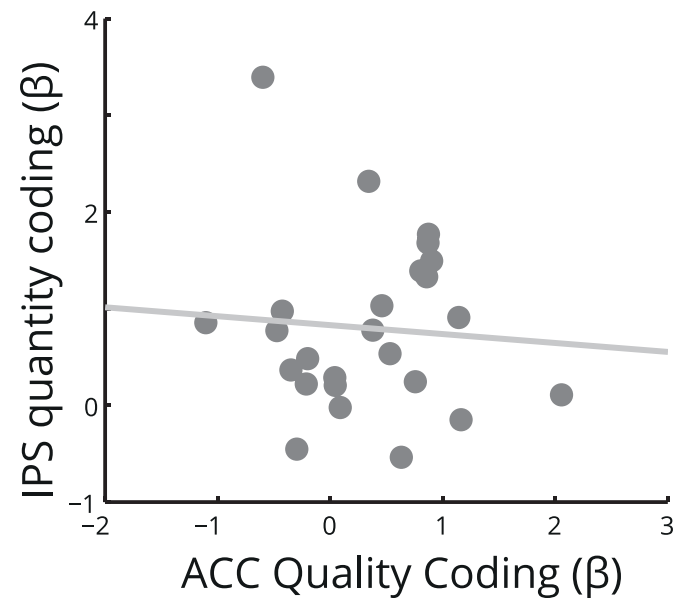

D

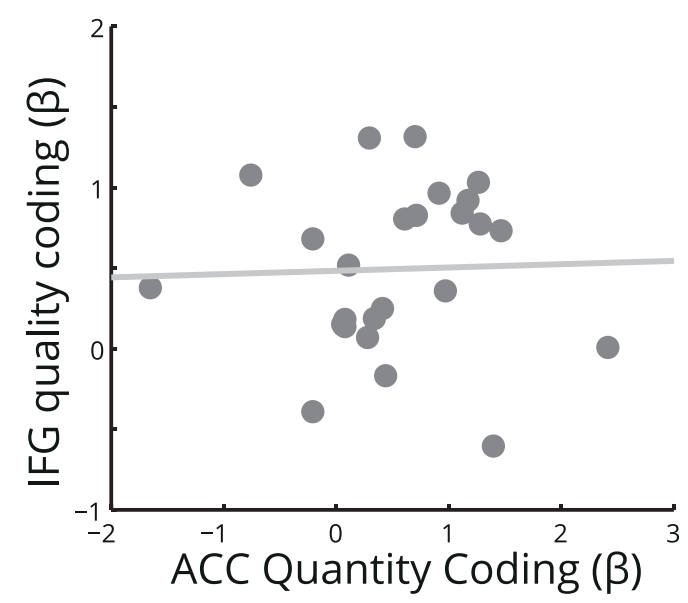

$\mathbf{F}$

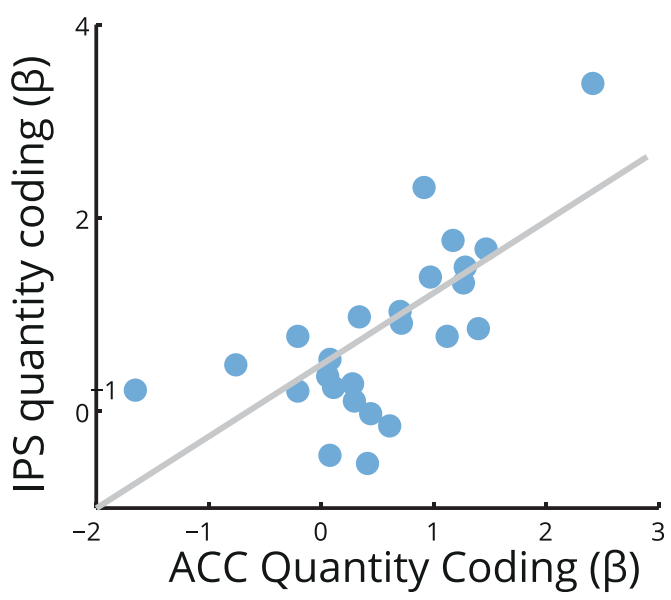

Figure 5. Computation of value from component parts in the anterior cingulate cortex. $A, 0$ verlapping effects of quality, quantity, and their interaction in the ACC. A conjunction analysis revealed overlapping representations of each component ( $p_{\text {uncorrected }}<0.05$; green) in the ACC, suggesting a nexus for the computation of value. A complementary analysis using an explicit representation of integrated value as a parametric modulator identified the same region ( $p_{\mathrm{FWE}-\text {-orrected }}<0.001$; turquoise). Time course displayed for illustration purposes. $\boldsymbol{B}$, Decision $>$ nondecision trials. The ACC also showed higher activity in trials upon which a decision was made compared with valuation trials (red), overlapping with the conjunction analysis identified in $\boldsymbol{A}$ (green). $\boldsymbol{C}$, Participants with stronger representations of quality in the IFG showed stronger representations of quality in the $\mathrm{ACC}(r=0.63, p<0.001)$. $\boldsymbol{D}$, Quality sensitivity in IFG was unrelated to quantity coding in $\mathrm{ACC}(r=$ $0.04, p=0.83)$. $\boldsymbol{E}$, Quantity sensitivity in IPS was unrelated to quality coding in ACC $(r=-0.14, p=0.50)$. $\boldsymbol{F}$, Participants with stronger representations of quantity in the IPS showed stronger representations of quantity in the $\mathrm{ACC}(r=0.68, p<0.001)$. Each point is one participant.

to ACC quantity $(r=0.04, p=0.83)$ and IPS quantity unrelated to ACC quality $(r=0.14, p=0.50)$ coding (Fig. $5 D, E)$. This specificity suggests that observed correlations reflect meaningful inter-regional relationships rather than correlated variance in signal-to-noise ratio between participants.

\section{Strength of neural quantity coding reflects} choice predictability

The degree to which subjects' choices were correctly predicted by our logistic regression varied from $69 \%$ to $92 \%$. We reasoned that stronger neural representations of value components should lead 

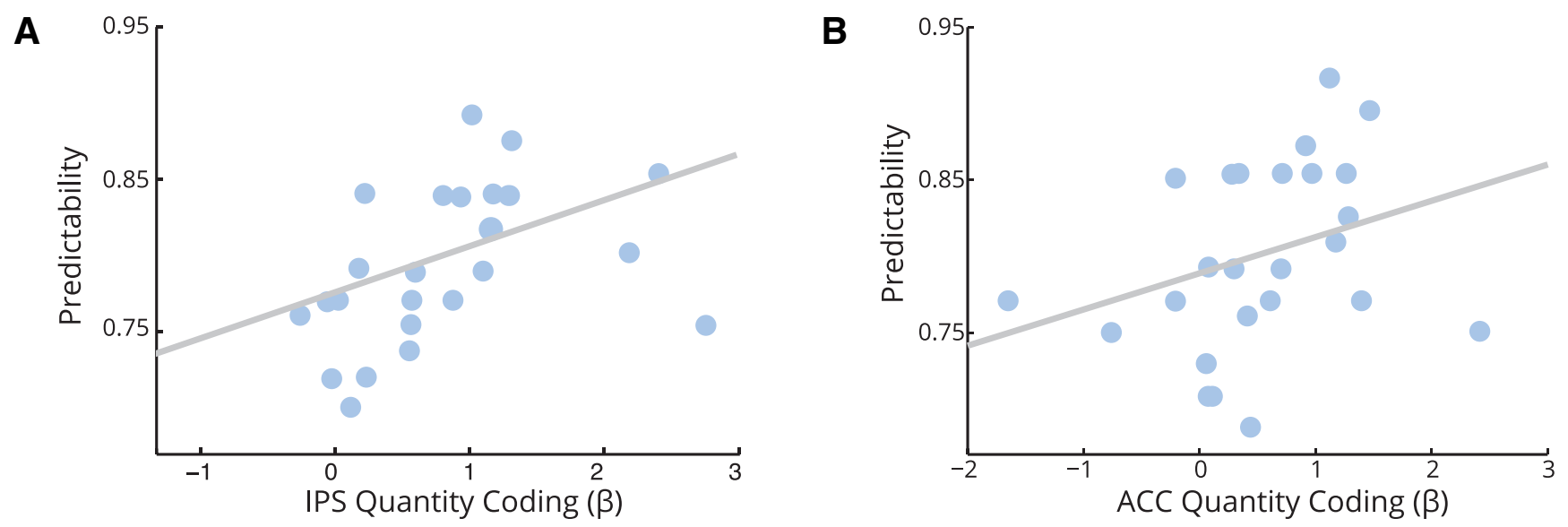

Figure 6. $A, B$, Neural quantity sensitivity relate to choice predictability. We found that coefficients for quantity in IPS $(\rho=0.60, p=0.002 ; A)$ and the ACC $(\rho=0.42, p=0.039 ; B)$ correlated with the predictability of participants' choices, as assessed by the ability of our logistic regression model to predict choice. Correlations with quality coding in the IFG $(\rho=0.41, p=0.104)$ and ACC $(\rho=0.30, p=0.142)$ were positive but not significant. Each point is one participant.

to more predictable choices. Using parameter estimates $(\beta$ values) extracted from our GLM analyses, we asked whether between-subject variability in $\beta$ values related to between-subject choice predictability. We found that the strength of neural correlations with quantity, but not quality, was associated with predictability of choice (Fig. 6). Mean $\beta$ values in both the IPS ( $\rho=$ $0.60, p=0.002)$ and ACC $(\rho=0.42, p=0.039)$ were positively correlated with choice predictability, suggesting that stronger neural representations of quantity correspond to more reliable choices. Correlations between predictability and quality coding in the IFG $(\rho=0.41, p=0.104)$ and ACC $(\rho=0.30, p=0.142)$ were also positive but did not reach significance, perhaps reflecting the greater range of values for quantity than quality or the potential impact of overtraining on giftcard quality. We observed no relationship between interaction coding in ACC and predictability $(\rho=-0.04, p=0.83)$. We note that after applying a conservative Bonferroni correction for the five comparisons we make here, only the effect in the IPS survives an adjusted threshold of $\alpha=0.01$.

Using the summed log-likelihood of choices according to the logistic regression model as an alternative measure of choice predictability yielded a consistent pattern of results, although the effect in the ACC was no longer significant at $\alpha=0.05$ (IPS: $\rho=0.55, p=$ 0.004; ACC: $\rho=0.35, p=0.089$ ). We did not observe any other correlations between the parameters of our logistic regression model for behavior and those of our GLMs for neural activity.

\section{Repetition suppression for integrated value in the ACC}

RS describes the phenomenon whereby repeated presentation of stimuli that are similar along some dimension evoke reduced activity in brain regions sensitive to that attribute (Grill-Spector et al., 2006). This is putatively due to a reduction in activity in neurons activated in both trials (Fig. 7). This provides a means to assay the neural overlap in the representation of two stimuli such as foods (Barron et al., 2013), faces (Loffler et al., 2005), or even agents (Garvert et al., 2015). This can reveal nonmonotonic codes invisible to traditional GLM approaches, such as tuned numerosity representations in the parietal cortex (Piazza et al., 2004, 2007; Jacob and Nieder, 2009). Since our task involved the calculation of value from information about quantity, we hypothesized that value representations in the ACC might show a similar form.

We asked whether RS provides additional evidence of value encoding in ACC. We constructed a GLM where we modeled the absolute difference in integrated value ( $\Delta$ IntegratedValue) between subsequent trials, as well as the Integrated Value on each trial. If neurons in a brain region are undergoing RS, aggregate activity as assayed by BOLD should covary with the absolute difference between trials (Barron et al., 2016). We found evidence for repetition suppression to value in dorsal ACC (peak MNI, $-3,3,51 ; t_{(24)}=3.86, p_{\mathrm{FWE}-\text { corrected }}=0.003$ ), just posterior to the activity related to monotonic encoding of integrated value (Fig. $7 B$ ). These activations were partially overlapping, such that the integrated value coding conjunction identified in Figure 5 showed effects of both $\Delta$ IntegratedValue and Integrated Value (Fig. $7 C$; $\Delta$ IntegratedValue: $t_{(24)}=2.48, p=0.020$; Integrated Value: $t=3.26, p=0.0034)$. Repetition suppression for integrated value was surprisingly widespread. We also observed repetition suppression for value bilaterally in the lingual gyrus (left: peak MNI, $-15,-45,-9 ; t_{(24)}=6.18, p_{\text {FWE-corrected }}<0.001$; right: peak MNI, $\left.15,-48,3 ; t_{(24)}=4.56, p_{\text {FWE-corrected }}<0.001\right)$, the right superior temporal sulcus (peak MNI, $63,-30,3 ; t_{(24)}=$ $\left.5.80, p_{\text {FWE-corrected }}<0.001\right)$, and bilaterally in the posterior insula (left: peak MNI, $-30,0,-3 ; t_{(24)}=5.00, p_{\text {FWE-corrected }}<0.001$; right: peak MNI, $33,-6,-12 ; t_{(24)}=3.60, p_{\text {FWE-corrected }}<$ $0.001)$.

\section{Discussion}

Value representations are often studied as monolithic entities. Indeed, considerable effort has been expended in identifying abstract behavioral and neural signatures of scalar value estimates. However, recent work suggests that during choice components of value compete at an attribute level to guide decisions (Hunt et al., 2014), emphasizing the importance of decomposing value into its constituent parts. Here we show that, in the absence of choice, integrated value correlates appear in the ACC, with component representations in the IFG (quality) and IPS (quantity; Figs. 5, 6). A distinct network appears to integrate the two, with posterior cingulate and superior temporal lobe activations corresponding to the interaction between quality and quantity (Fig. 4). A more posterior region of the ACC displays repetition suppression to integrated value (Fig. 7).

\section{Correlates of quality and quantity in the brain}

Bilateral IFG activity scaled with the quality of the giftcard presented on each trial (Fig. 4A). This was unexpected, given the 
A
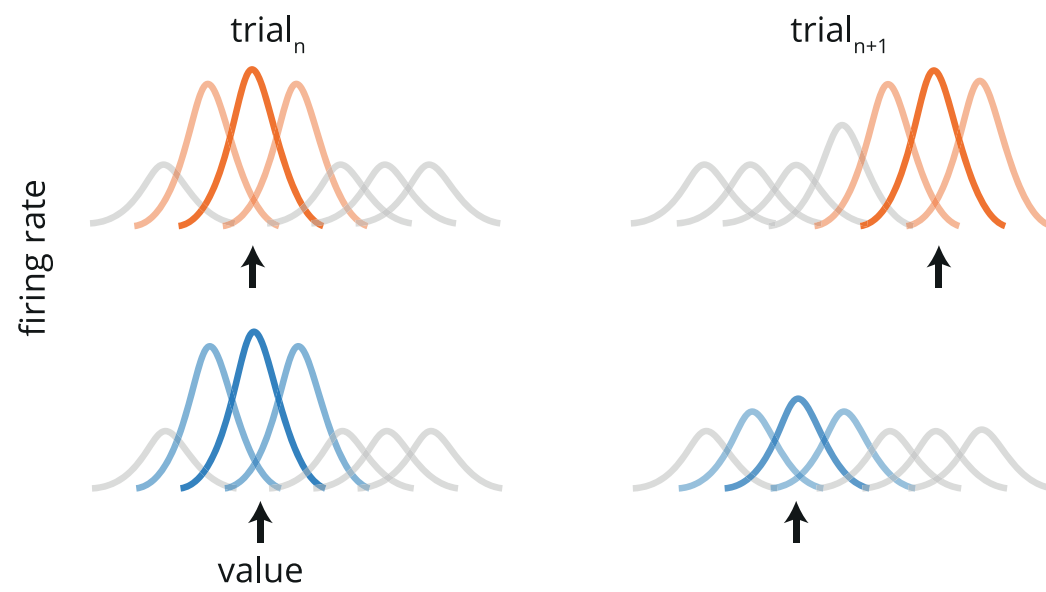

B

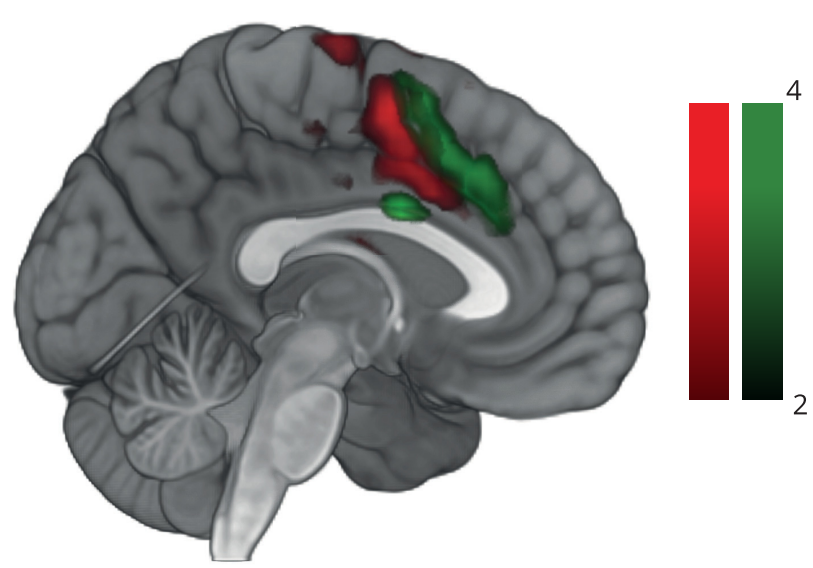

C

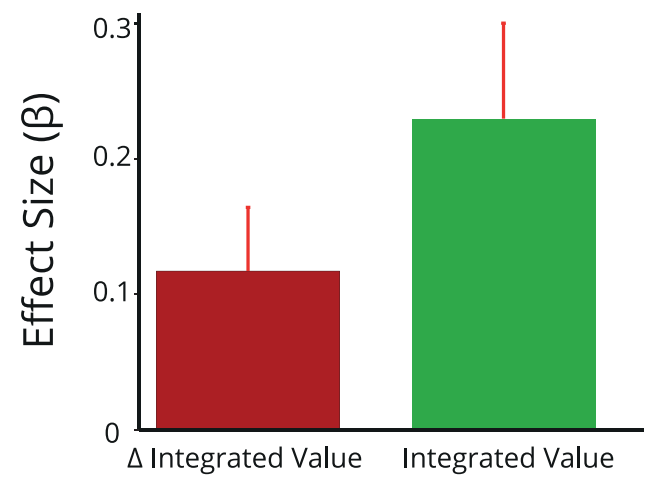

Figure 7. Repetition suppression for value in the anterior cingulate cortex. A, Repetition suppression analysis logic. We hypothesize a population of neurons tuned to value where the different neurons have overlapping tuning curves spanning the range of values presented. Black arrows denote stimulus value for that trial. If consecutive trials activate nonoverlapping populations of neurons, evoked responses for each stimulus are similarly high on each trial (top panel in orange). However, repeated presentation of the same stimulus produces repeated activation of the same neurons on consecutive trials, leading to a reduction in the neural response (bottom panel in blue). Summation over all neurons in the population (as in the BOLD signal measured in fMRI), leads to higher activity when consecutive stimuli activate unique subsets of neurons (top panel) than when consecutively activated populations overlap (bottom panel). Predicted BOLD activity is thus proportional to the absolute difference in value between consecutive trials. $\boldsymbol{B}$, Evidence for multiple forms of value coding in the cingulate. We examined cingulate representations of repetition suppression to integrated value (change in value from trial $n-1$ to trial $n, \Delta$ IntegratedValue; green), and monotonic encoding of integrated value (a standard parametric modulator approach; red). Voxels sensitive to repetition suppression were more posterior, with monotonic encoding stronger in anterior voxels. C, The ACC region identified in the conjunction analysis (Fig. $5 \mathrm{~A}$ ) also shows repetition suppression in the integrated value. We extracted mean parameter estimates for $\Delta$ IntegratedValue and for integrated value from the voxels identified in the conjunction analysis. Both were positive on average ( $\Delta$ IntegratedValue: $t_{(24)}=2.48, p=0.020$; Integrated Value: $t=3.26, p=0.0034$ ). Error bars represent the SEM across participants.

scarcity of reports of IFG involvement in value-based decisionmaking (but see Rogers et al., 1999; Zysset et al., 2006; Liljeholm et al., 2011). A priori, the OFC might represent a more promising candidate for the representation of stimulus quality. However, representations in the OFC appear to be particularly entangled with stimulus identity (Padoa-Schioppa and Assad, 2008; Noonan et al., 2011; Barron et al., 2013; Klein-Flügge et al., 2013; McNamee et al., 2013; Howard et al., 2015), potentially reflecting the central role of the OFC in providing an internal model of the world (Wilson et al., 2014). For instance, Padoa-Schioppa and Assad (2008) describe OFC cells that respond specifically to one juice or another, which they describe as reflecting the taste of a given juice. This encoding of juice identity is distinct from the reward quality, and no study has reported OFC unit responses that reflect quality alone (i.e., the preference ordering of different stimuli), while being insensitive to quantity. It seems, therefore, that the OFC is particularly interested in tracking relationships between specific rewards and their predictors (Noonan et al., 2011; Takahashi et al., 2013; Stalnaker et al., 2014, 2015; Lopatina et al., 2015; Lucantonio et al., 2015; Boorman et al., 2016), rather than estimating stimulus quality per se. Furthermore, a recent study (Schuck et al., 2016) found that OFC exclusively represented hidden variables related to the current state. The lack of OFC involvement in our task is likely to reflect the static and transparent relationship between stimuli and outcomes in our experiment.

The involvement of the IFG in the representation of stimulus quality is consistent with the semantic nature of the giftcard stimuli we used. IFG is commonly activated in lexical tasks (Price, 2012), with left hemisphere lesions to this area producing impairments in language production and comprehension. In one of the few studies attempting to parse value into distinct components, Lim et al. (2013) offered participants T-shirts that varied in their esthetic and semantic properties. They found correlations with esthetic value in the fusiform gyrus and semantic value in the superior temporal gyrus, while ventromedial PFC (vmPFC) activity correlated with the value of both attributes. This suggests that the extraction of quality may occur in concert across brain areas specialized for the analysis of distinct stimulus features, in the same way that feedforward models of visual inputs eventually 
produce value estimates in deep reinforcement learning networks (Mnih et al., 2015; Silver et al., 2016). This suggests that a representation of stimulus quality in IFG may be specific to semantically rich stimuli, such as those used here.

Conversely, our observation of quantity coding in the IPS (Fig. 4B) is predicted from the literature (Nieder, 2016). A wide variety of animals show an ability to make ethologically relevant decisions using number, from lions (McComb et al., 1994) to crows (Rahman et al., 2014). Even newborn chicks are capable of tracking the number of an imprinted object that is placed behind a screen (Rugani et al., 2009). In macaques, such judgments rely upon a network of frontal and parietal regions containing neurons tuned to different numbers, including the number zero (Nieder et al., 2002; Nieder and Miller, 2004; Ramirez-Cardenas et al., 2016).

Studies in humans have made use of model-based decoding analyses (Harvey et al., 2013) and repetition suppression designs (Piazza et al., 2004, 2007; Jacob and Nieder, 2009) to provide evidence that similar tuning curves for number exist in the human IPS. Our results imply that the same IPS circuitry subserves number representation in value computation. This is consistent with the recent observation that when number and value are decorrelated, the IPS tracks quantity and not value (Kanayet et al., 2014). This serves to clarify the role of parietal cortices in value-based decision-making, suggesting that when financial stimuli are used (Ballard and Knutson, 2009; Clithero et al., 2009; Chau et al., 2014), evaluation occurs within a financial framework (e.g., the BDM auction; Plassmann et al., 2007; Medic et al., 2014), or, if stimuli merely differ in magnitude (Louie et al., 2011), parietal responses to quantity may be misconstrued as representing value or its comparison. Conversely, we find that the IPS specifically represents the quantity of an available option, and that the strength of numerical representations in IPS correlates both with choice predictability and ACC quantity coding. This is consistent with neurons in IPS contributing to the representation of stimulus value in the ACC, and this latter representation subsequently being used to guide choice. However, it is unclear why choice predictability is so much more strongly linked to quantity coding than it is to quality coding (in IFG and ACC) and to interaction coding (in the ACC). We suspect that the greater range of quantities than qualities in our experiment may have increased our power to observe relationships with quantity coding, but this remains an open question.

\section{The role of the ACC in evaluation}

We found that activity in the ACC was consistent with the representation of integrated value. ACC showed a positive correlation with integrated value. Even after accounting for the effects of quality and quality, ACC tracked the interaction term characteristic of integrated value in this task (Figs. 5C, 6A). Beckmann et al. (2009) parcellated the cingulate cortex according to connectivity. The region we identify corresponds to their region 4 , which shows strong connectivity to dorsolateral prefrontal cortex, and is commonly implicated in value-based tasks. The region showing repetition suppression effects may be more situated in their region 5, which has a higher connectivity to the parietal cortex. This raises the possibility that the repetition suppression we observe is inherited from tuned numerical representations in parietal cortex (Nieder and Miller, 2004).

The ACC is frequently identified in both human (Bush et al., 2002; Kolling et al., 2012; Boorman et al., 2013) and animal experiments (Seo and Lee, 2007; Hayden et al., 2009; Hayden and Platt, 2010; Kennerley et al., 2011; Cai and Padoa-Schioppa,
2012) of value-based choice. The more dorsal region in which we find signatures of integrated value is associated with tasks wherein participants assign value to actions (Beckmann et al., 2009). This is the case in our experiment, since giftcards were displayed either on the left-hand or the right-hand side of the screen, such that assessing the value of a particular giftcard was the same as assessing the value of a left/right button press. Dorsal ACC appears to be particularly engaged by foraging type tasks, in which the pertinent comparisons are between options presented sequentially (Seo and Lee, 2007; Kolling et al., 2012; Boorman et al., 2013). We further note that since the positive value correlations we observe in the ACC are recorded in the absence of choice, they cannot be explained as a function of choice difficulty and are more consistent with a proposed role in sequential foraging decisions (Kolling et al., 2016).

We did not observe value-related activity in the vmPFC, the part of the cortex most frequently associated with valuation (Rushworth and Behrens, 2008), or in the ventral striatum. This agrees with recent observations suggesting that sequential (Hunt et al., 2013) or time-limited (Jocham et al., 2014) choices do not engage vmPFC. Indeed, a growing body of evidence suggests that the ACC is particularly involved when subjects make sequential, foraging-type decisions, which are characterized by an evaluation of whether to engage or not (Kolling et al., 2012, 2016). Conversely, whether evaluation alone effectively engages vmPFC is unclear. Although early reports suggested that the vmPFC was part of an automatic valuation system (Lebreton et al., 2009), recent work suggests otherwise (Grueschow et al., 2015). The few studies that report value-related activity in macaque vmPFC do so in the context of free viewing (Strait et al., 2014; Abitbol et al., 2015), raising the possibility that the vmPFC is particularly engaged when values are compared via repeated eye movements (Krajbich et al., 2010). The observation that vmPFC is crucial for episodic memory and imagination (Hassabis and Maguire, 2009; Benoit et al., 2014), and a predominance of saccade-frequency theta oscillations in mPFC (Paz et al., 2008; Adhikari et al., 2010) hints at a more general role for the vmPFC in mediating a shortterm plasticity allowing features - of a scene, an episode, or a choice-to be integrated over several seconds. This might explain why our task, which required participants to evaluate a single stimulus at a single location, did not modulate vmPFC activity.

Our finding that the cingulate cortex integrates information about quality and quantity to form a multiplicative value representation of the current stimulus is also interesting in light of a literature implicating the cingulate in the representation of values associated with "model-based" cognition (Wunderlich et al., 2012; Doll et al., 2015). This describes flexible computation of value associated with a certain stimulus, and is typically contrasted with "model-free" cognition, in which stimulus or action values are cached and updated only through repeated experience (Dolan and Dayan, 2013). The multiplication of quantity and quality that we observe in the ACC is consistent with the idea that the cingulate provides a model that produces estimates of quantities relevant to behavior (O'Reilly et al., 2013; Economides et al., 2014; Kolling et al., 2014). In our case, utility was maximized by combining quality and quantity in a multiplicative manner, and this is what the ACC appears to do, in a manner that reflects the coding of quality and quantity in the frontal and parietal lobes respectively (Fig. 5C,F).

Our design also enabled us to perform a repetition suppression analysis, allowing us to reveal coding schemes hidden to conventional BOLD analyses. We found that parts of the cingulate cortex displayed repetition suppression to integrated value, 
with activity that scaled with the absolute difference in value between trials (Fig. 7A). This region was posterior to the peak activity associated with monotonic integrated value, extending into the area identified in the conjunction analysis of quality, quantity, and their interaction (Fig. 7B). Although the precedent from the numerosity-coding literature is to suppose that RS results of this kind provide positive evidence of nonmonotonic tuning (Piazza et al., 2004, 2007; Ansari and Dhital, 2006; Jacob and Nieder, 2009), we are cognizant that such repetition suppression effects are not an unambiguous signature of nonmonotonic codes. In modeling work reported previously, we observe that repetition suppression effects such as the ones we observe here can result from mixed linear codes combined with divisive adaptation. Furthermore, given the role of the ACC in comparing option values over time (Kolling et al., 2012, 2014), the observed relationship with variance in value from trial to trial could reflect a cognitively meaningful surprise signal, potentially related to environmental volatility (Behrens et al., 2007). Recent work observes just such a signal in a biophysically plausible model of reward learning, in which learning is adapted to volatility via metaplasticity (Farashahi et al., 2017).

To conclude, we find that a distributed network comprising the intraparietal sulcus, inferior frontal gyrus, and posterior cingulate and superior temporal sulcus contribute to the computation of integrated value in the ACC. The strength of signals in the ACC reflected the degree to which they were represented in brain areas coding for quality (IFG) and quantity (IPS), and stronger brain correlations with quantity were associated with more predictable choices. We further demonstrate that parts of the ACC also show repetition suppression to integrated value, which is consistent with the idea that tuning for value is nonmonotonic in parts of the cortex. Our findings demonstrate how value is assembled from its component parts and emphasize the potential for repetition suppression as an assay of a populationencoding scheme.

\section{References}

Abitbol R, Lebreton M, Hollard G, Richmond BJ, Bouret S, Pessiglione M (2015) Neural mechanisms underlying contextual dependency of subjective values: converging evidence from monkeys and humans. J Neurosci 35:2308-2320. CrossRef Medline

Adhikari A, Topiwala MA, Gordon JA (2010) Synchronized activity between the ventral hippocampus and the medial prefrontal cortex during anxiety. Neuron 65:257-269. CrossRef Medline

Ansari D, Dhital B (2006) Age-related changes in the activation of the intraparietal sulcus during nonsymbolic magnitude processing: an eventrelated functional magnetic resonance imaging study. J Cogn Neurosci 18:1820-1828. CrossRef Medline

Ashburner J (2007) A fast diffeomorphic image registration algorithm. Neuroimage 38:95-113. CrossRef Medline

Ballard K, Knutson B (2009) Dissociable neural representations of future reward magnitude and delay during temporal discounting. Neuroimage 45:143-150. CrossRef Medline

Barron HC, Garvert MM, Behrens TEJ (2016) Repetition suppression: a means to index neural representations using BOLD? Philos Trans R Soc Lond B, Biol Sci 371:20150355. CrossRef Medline

Barron HC, Dolan RJ, Behrens TE (2013) Online evaluation of novel choices by simultaneous representation of multiple memories. Nat Neurosci 16:1492-1498. CrossRef Medline

Becker GM, DeGroot MH, Marschak J (1964) Measuring utility by a singleresponse sequential method. Behav Sci 9:226-232. CrossRef Medline

Beckmann M, Johansen-Berg H, Rushworth MF (2009) Connectivity-based parcellation of human cingulate cortex and its relation to functional specialization. J Neurosci 29:1175-1190. CrossRef Medline

Behrens TE, Woolrich MW, Walton ME, Rushworth MF (2007) Learning the value of information in an uncertain world. Nat Neurosci 10:12141221. CrossRef Medline
Benoit RG, Szpunar KK, Schacter DL (2014) Ventromedial prefrontal cortex supports affective future simulation by integrating distributed knowledge. Proc Natl Acad Sci U S A 111:16550-16555. CrossRef Medline

Boorman ED, Rushworth MF, Behrens TE (2013) Ventromedial prefrontal and anterior cingulate cortex adopt choice and default reference frames during sequential multi-alternative choice. J Neurosci 33:2242-2253. CrossRef Medline

Boorman ED, Rajendran VG, O'Reilly JX, Behrens TE (2016) Two anatomically and computationally distinct learning signals predict changes to stimulus-outcome associations in hippocampus. Neuron 89:1343-1354. CrossRef Medline

Bush G, Vogt BA, Holmes J, Dale AM, Greve D, Jenike MA, Rosen BR (2002) Dorsal anterior cingulate cortex: a role in reward-based decision making. Proc Natl Acad Sci U S A 99:523-528. CrossRef Medline

Cai X, Padoa-Schioppa C (2012) Neuronal encoding of subjective value in dorsal and ventral anterior cingulate cortex. J Neurosci 32:3791-3808. CrossRef Medline

Chau BK, Kolling N, Hunt LT, Walton ME, Rushworth MF (2014) A neural mechanism underlying failure of optimal choice with multiple alternatives. Nat Neurosci 17:463-470. CrossRef Medline

Cisek P, Kalaska JF (2010) Neural mechanisms for interacting with a world full of action choices. Annu Rev Neurosci 33:269-298. CrossRef Medline

Clithero JA, Carter RM, Huettel SA (2009) Local pattern classification differentiates processes of economic valuation. Neuroimage 45:1329-1338. CrossRef Medline

Deichmann R, Schwarzbauer C, Turner R (2004) Optimisation of the 3D MDEFT sequence for anatomical brain imaging: technical implications at 1.5 and 3 T. Neuroimage 21:757-767. CrossRef Medline

De Martino B, Fleming SM, Garrett N, Dolan RJ (2013) Confidence in value-based choice. Nat Neurosci 16:105-110. CrossRef Medline

Dolan RJ, Dayan P (2013) Goals and habits in the brain. Neuron 80:312325. CrossRef Medline

Doll BB, Duncan KD, Simon DA, Shohamy D, Daw ND (2015) Modelbased choices involve prospective neural activity. Nat Neurosci 18:767772. CrossRef Medline

Economides M, Guitart-Masip M, Kurth-Nelson Z, Dolan RJ (2014) Anterior cingulate cortex instigates adaptive switches in choice by integrating immediate and delayed components of value in ventromedial prefrontal cortex. J Neurosci 34:3340-3349. CrossRef Medline

Farashahi S, Donahue CH, Khorsand P, Seo H, Lee D, Soltani A (2017) Metaplasticity as a neural substrate for adaptive learning and choice under uncertainty. Neuron 94:401-414.e6. CrossRef Medline

FitzGerald TH, Seymour B, Dolan RJ (2009) The role of human orbitofrontal cortex in value comparison for incommensurable objects. J Neurosci 29:8388-8395. CrossRef Medline

Friston KJ, Holmes AP, Price CJ, Büchel C, Worsley KJ (1999) Multisubject fMRI studies and conjunction analyses. Neuroimage 10:385-396. CrossRef Medline

Garvert MM, Moutoussis M, Kurth-Nelson Z, Behrens TE, Dolan RJ (2015) Learning-induced plasticity in medial prefrontal cortex predicts preference malleability. Neuron 85:418-428. CrossRef Medline

Grill-Spector K, Henson R, Martin A (2006) Repetition and the brain: neural models of stimulus-specific effects. Trends Cogn Sci 10:14-23. CrossRef Medline

Grueschow M, Polania R, Hare TA, Ruff CC (2015) Automatic versus choice-dependent value representations in the human brain. Neuron 85: 874-885. CrossRef Medline

Hare TA, Schultz W, Camerer CF, O’Doherty JP, Rangel A (2011) Transformation of stimulus value signals into motor commands during simple choice. Proc Natl Acad Sci U S A 108:18120-18125. CrossRef Medline

Harvey BM, Klein BP, Petridou N, Dumoulin SO (2013) Topographic representation of numerosity in the human parietal cortex. Science 341: 1123-1126. CrossRef Medline

Hassabis D, Maguire EA (2009) The construction system of the brain. Philos Trans R Soc Lond B Biol Sci 364:1263-1271. CrossRef Medline

Hayden BY, Platt ML (2010) Neurons in anterior cingulate cortex multiplex information about reward and action. J Neurosci 30:3339-3346. CrossRef Medline

Hayden BY, Pearson JM, Platt ML (2009) Fictive reward signals in the anterior cingulate cortex. Science 324:948-950. CrossRef Medline

Hayden BY, Pearson JM, Platt ML (2011) Neuronal basis of sequential for- 
aging decisions in a patchy environment. Nat Neurosci 14:933-939. CrossRef Medline

Howard JD, Gottfried JA, Tobler PN, Kahnt T (2015) Identity-specific coding of future rewards in the human orbitofrontal cortex. Proc Natl Acad Sci U S A 112:5195-5200. CrossRef Medline

Hunt LT, Kolling N, Soltani A, Woolrich MW, Rushworth MF, Behrens TE (2012) Mechanisms underlying cortical activity during value-guided choice. Nat Neurosci 15:470-476, S1-S3. CrossRef Medline

Hunt LT, Dolan RJ, Behrens TE (2014) Hierarchical competitions subserving multi-attribute choice. Nat Neurosci 17:1613-1622. CrossRef Medline

Hunt LT, Woolrich MW, Rushworth MFS, Behrens TEJ (2013) Trial-Type Dependent Frames of Reference for Value Comparison. PLOS Computational Biology 9: e1003225. CrossRef

Hunt LT, Behrens TE, Hosokawa T, Wallis JD, Kennerley SW (2015) Capturing the temporal evolution of choice across prefrontal cortex. Elife 4:e11945. CrossRef Medline

Hutton C, Josephs O, Stadler J, Featherstone E, Reid A, Speck O, Bernarding J, Weiskopf N (2011) The impact of physiological noise correction on fMRI at 7 T. Neuroimage 57:101-112. CrossRef Medline

Jacob SN, Nieder A (2009) Notation-independent representation of fractions in the human parietal cortex. J Neurosci 29:4652-4657. CrossRef Medline

Jocham G, Furlong PM, Kröger IL, Kahn MC, Hunt LT, Behrens TE (2014) Dissociable contributions of ventromedial prefrontal and posterior parietal cortex to value-guided choice. Neuroimage 100:498-506. CrossRef Medline

Kable JW, Glimcher PW (2007) The neural correlates of subjective value during intertemporal choice. Nat Neurosci 10:1625-1633. CrossRef Medline

Kahnt T, Heinzle J, Park SQ, Haynes JD (2011) Decoding the formation of reward predictions across learning. J Neurosci 31:14624-14630. CrossRef Medline

Kanayet FJ, Opfer JE, Cunningham WA (2014) The value of numbers in economic rewards. Psychol Sci 25:1534-1545. CrossRef Medline

Kennerley SW, Behrens TE, Wallis JD (2011) Double dissociation of value computations in orbitofrontal and anterior cingulate neurons. Nat Neurosci 14:1581-1589. CrossRef Medline

Klein-Flügge MC, Barron HC, Brodersen KH, Dolan RJ, Behrens TE (2013) Segregated encoding of reward-identity and stimulus-reward associations in human orbitofrontal cortex. J Neurosci 33:3202-3211. CrossRef Medline

Knutson B, Rick S, Wimmer GE, Prelec D, Loewenstein G (2007) Neural predictors of purchases. Neuron 53:147-156. CrossRef Medline

Kolling N, Behrens TE, Mars RB, Rushworth MF (2012) Neural mechanisms of foraging. Science 336:95-98. CrossRef Medline

Kolling N, Wittmann M, Rushworth MFS (2014) Multiple neural mechanisms of decision making and their competition under changing risk pressure. Neuron 81:1190-1202. CrossRef Medline

Kolling N, Behrens T, Wittmann MK, Rushworth M (2016) Multiple signals in anterior cingulate cortex. Curr Opin Neurobiol 37:36-43. CrossRef Medline

Krajbich I, Armel C, Rangel A (2010) Visual fixations and the computation and comparison of value in simple choice. Nat Neurosci 13:1292-1298. CrossRef Medline

Lebreton M, Jorge S, Michel V, Thirion B, Pessiglione M (2009) An automatic valuation system in the human brain: evidence from functional neuroimaging. Neuron 64:431-439. CrossRef Medline

Levy I, Lazzaro SC, Rutledge RB, Glimcher PW (2011) Choice from nonchoice: predicting consumer preferences from blood oxygenation leveldependent signals obtained during passive viewing. J Neurosci 31:118125. CrossRef Medline

Liljeholm M, Tricomi E, O’Doherty JP, Balleine BW (2011) Neural correlates of instrumental contingency learning: differential effects of actionreward conjunction and disjunction. J Neurosci 31:2474-2480. CrossRef Medline

Lim SL, O’Doherty JP, Rangel A (2013) Stimulus value signals in ventromedial PFC reflect the integration of attribute value signals computed in fusiform gyrus and posterior superior temporal gyrus. J Neurosci 33: 8729-8741. CrossRef Medline

Loffler G, Yourganov G, Wilkinson F, Wilson HR (2005) fMRI evidence for the neural representation of faces. Nat Neurosci 8:1386-1390. CrossRef Medline

Lopatina N, McDannald MA, Styer CV, Sadacca BF, Cheer JF, Schoenbaum G (2015) Lateral orbitofrontal neurons acquire responses to upshifted, downshifted, or blocked cues during unblocking. Elife 4:e11299. CrossRef Medline

Louie K, Grattan LE, Glimcher PW (2011) Reward value-based gain control: divisive normalization in parietal cortex. J Neurosci 31:10627-10639. CrossRef Medline

Lucantonio F, Gardner MP, Mirenzi A, Newman LE, Takahashi YK, Schoenbaum G (2015) Neural estimates of imagined outcomes in basolateral amygdala depend on orbitofrontal cortex. J Neurosci 35:16521-16530. CrossRef Medline

McComb K, Packer C, Pusey A (1994) Roaring and numerical assessment in contests between groups of female lions, Panthera leo. Anim Behav 47: 379-387. CrossRef

McNamee D, Rangel A, O’Doherty JP (2013) Category-dependent and category-independent goal-value codes in human ventromedial prefrontal cortex. Nat Neurosci 16:479-485. CrossRef Medline

Medic N, Ziauddeen H, Vestergaard MD, Henning E, Schultz W, Farooqi IS, Fletcher PC (2014) Dopamine modulates the neural representation of subjective value of food in hungry subjects. J Neurosci 34:16856-16864. CrossRef Medline

Mnih V, Kavukcuoglu K, Silver D, Rusu AA, Veness J, Bellemare MG, Graves A, Riedmiller M, Fidjeland AK, Ostrovski G, Petersen S, Beattie C, Sadik A, Antonoglou I, King H, Kumaran D, Wierstra D, Legg S, Hassabis D (2015) Human-level control through deep reinforcement learning. Nature 518:529-533. CrossRef Medline

Montague PR, Berns GS (2002) Neural economics and the biological substrates of valuation. Neuron 36:265-284. CrossRef Medline

Nieder A (2016) The neuronal code for number. Nat Rev Neurosci 17:366382. CrossRef Medline

Nieder A, Miller EK (2004) A parieto-frontal network for visual numerical information in the monkey. Proc Natl Acad Sci U S A 101:7457-7462. CrossRef Medline

Nieder A, Freedman DJ, Miller EK (2002) Representation of the quantity of visual items in the primate prefrontal cortex. Science 297:1708-1711. CrossRef Medline

Noonan MP, Mars RB, Rushworth MF (2011) Distinct roles of three frontal cortical areas in reward-guided behavior. J Neurosci 31:14399-14412. CrossRef Medline

O’Doherty JP (2014) The problem with value. Neurosci Biobehav Rev 43: 259-268. CrossRef Medline

O’Doherty J, Kringelbach ML, Rolls ET, Hornak J, Andrews C (2001) Abstract reward and punishment representations in the human orbitofrontal cortex. Nat Neurosci 4:95-102. CrossRef Medline

O'Reilly JX, Schüffelgen U, Cuell SF, Behrens TE, Mars RB, Rushworth MF (2013) Dissociable effects of surprise and model update in parietal and anterior cingulate cortex. Proc Natl Acad Sci U S A 110:E3660-E3669. CrossRef Medline

Padoa-Schioppa C (2011) Neurobiology of economic choice: a good-based model. Annu Rev Neurosci 34:333-359. CrossRef Medline

Padoa-Schioppa C, Assad JA (2006) Neurons in the orbitofrontal cortex encode economic value. Nature 441:223-226. CrossRef Medline

Padoa-Schioppa C, Assad JA (2008) The representation of economic value in the orbitofrontal cortex is invariant for changes of menu. Nat Neurosci 11:95-102. CrossRef Medline

Padoa-Schioppa C, Schoenbaum G (2015) Dialogue on economic choice, learning theory, and neuronal representations. Curr Opin Behav Sci 5:16-23. CrossRef Medline

Paz R, Bauer EP, Paré D (2008) Theta synchronizes the activity of medial prefrontal neurons during learning. Learn Mem 15:524-531. CrossRef Medline

Pessiglione M, Seymour B, Flandin G, Dolan RJ, Frith CD (2006) Dopamine-dependent prediction errors underpin reward-seeking behaviour in humans. Nature 442:1042-1045. CrossRef Medline

Piazza M, Izard V, Pinel P, Le Bihan D, Dehaene S (2004) Tuning curves for approximate numerosity in the human intraparietal sulcus. Neuron 44: 547-555. CrossRef Medline

Piazza M, Pinel P, Le Bihan D, Dehaene S (2007) A magnitude code common to numerosities and number symbols in human intraparietal cortex. Neuron 53:293-305. CrossRef Medline

Pinel P, Piazza M, Le Bihan D, Dehaene S (2004) Distributed and overlapping cerebral representations of number, size, and luminance during comparative judgments. Neuron 41:983-993. CrossRef Medline

Plassmann H, O'Doherty J, Rangel A (2007) Orbitofrontal cortex encodes 
willingness to pay in everyday economic transactions. J Neurosci 27: 9984-9988. CrossRef Medline

Polanía R, Krajbich I, Grueschow M, Ruff CC (2014) Neural oscillations and synchronization differentially support evidence accumulation in perceptual and value-based decision making. Neuron 82:709-720. CrossRef Medline

Price CJ (2012) A review and synthesis of the first 20years of PET and fMRI studies of heard speech, spoken language and reading. Neuroimage 62: 816-847. CrossRef Medline

Rahman NA, Fadzly N, Dzakwan NM, Zulkifli NH (2014) The numerical competency of two bird species (Corvus splendens and acridotheres tristis). Trop Life Sci Res 25:95-103. Medline

Ramirez-Cardenas A, Moskaleva M, Nieder A (2016) Neuronal representation of numerosity zero in the primate parieto-frontal number network. Curr Biol 26:1285-1294. CrossRef Medline

Rogers RD, Owen AM, Middleton HC, Williams EJ, Pickard JD, Sahakian BJ, Robbins TW (1999) Choosing between small, likely rewards and large, unlikely rewards activates inferior and orbital prefrontal cortex. J Neurosci 19:9029-9038. CrossRef Medline

Rugani R, Fontanari L, Simoni E, Regolin L, Vallortigara G (2009) Arithmetic in newborn chicks. Proc Biol Sci 276:2451-2460. CrossRef Medline

Rushworth MF, Behrens TE (2008) Choice, uncertainty and value in prefrontal and cingulate cortex. Nat Neurosci 11:389-397. CrossRef Medline

Rutledge RB, Dean M, Caplin A, Glimcher PW (2010) Testing the reward prediction error hypothesis with an axiomatic model. J Neurosci 30: 13525-13536. CrossRef Medline

Schuck NW, Cai MB, Wilson RC, Niv Y (2016) Human orbitofrontal cortex represents a cognitive map of state space. Neuron 91:1402-1412. CrossRef Medline

Schultz W, Dayan P, Montague PR (1997) A neural substrate of prediction and reward. Science 275:1593-1599. CrossRef Medline

Seo H, Lee D (2007) Temporal filtering of reward signals in the dorsal anterior cingulate cortex during a mixed-strategy game. J Neurosci 27:83668377. CrossRef Medline

Silver D, Huang A, Maddison CJ, Guez A, Sifre L, van den Driessche G, Schrittwieser J, Antonoglou I, Panneershelvam V, Lanctot M, Dieleman S, Grewe D, Nham J, Kalchbrenner N, Sutskever I, Lillicrap T, Leach M,
Kavukcuoglu K, Graepel T, Hassabis D (2016) Mastering the game of go with deep neural networks and tree search. Nature 529:484-489. CrossRef Medline

Stalnaker TA, Cooch NK, McDannald MA, Liu TL, Wied H, Schoenbaum G (2014) Orbitofrontal neurons infer the value and identity of predicted outcomes. Nat Commun 5:3926. CrossRef Medline

Stalnaker TA, Cooch NK, Schoenbaum G (2015) What the orbitofrontal cortex does not do. Nat Neurosci 18:620-627. CrossRef Medline

Strait CE, Blanchard TC, Hayden BY (2014) Reward value comparison via mutual inhibition in ventromedial prefrontal cortex. Neuron 82:13571366. CrossRef Medline

Summerfield C, Trittschuh EH, Monti JM, Mesulam MM, Egner T (2008) Neural repetition suppression reflects fulfilled perceptual expectations. Nat Neurosci 11:1004-1006. CrossRef Medline

Sutton RS, Barto AG (1998) Reinforcement learning: an introduction. Cambridge, MA: MIT.

Takahashi YK, Chang CY, Lucantonio F, Haney RZ, Berg BA, Yau HJ, Bonci A, Schoenbaum G (2013) Neural estimates of imagined outcomes in the orbitofrontal cortex drive behavior and learning. Neuron 80:507-518. CrossRef Medline

Wang XJ (2008) Decision making in recurrent neuronal circuits. Neuron 60:215-234. CrossRef Medline

Weiskopf N, Hutton C, Josephs O, Deichmann R (2006) Optimal EPI parameters for reduction of susceptibility-induced BOLD sensitivity losses: a whole-brain analysis at $3 \mathrm{~T}$ and $1.5 \mathrm{~T}$. Neuroimage 33:493-504. CrossRef Medline

Wilson RC, Takahashi YK, Schoenbaum G, Niv Y (2014) Orbitofrontal cortex as a cognitive map of task space. Neuron 81:267-279. CrossRef Medline

Wunderlich K, Dayan P, Dolan RJ (2012) Mapping value based planning and extensively trained choice in the human brain. Nat Neurosci 15:786791. CrossRef Medline

Xie J, Padoa-Schioppa C (2016) Neuronal remapping and circuit persistence in economic decisions. Nat Neurosci 19:855-861. CrossRef Medline

Zysset S, Wendt CS, Volz KG, Neumann J, Huber O, von Cramon DY (2006) The neural implementation of multi-attribute decision making: a parametric fMRI study with human subjects. Neuroimage 31:1380-1388. CrossRef Medline 